\title{
CARGESE LECTURES ON EXTRA-DIMENSIONS
}

\author{
Riccardo RATTAZZI \\ Department of Physics, Theory Division \\ CERN, CH-1211 Geneva 23, Switzerland \\ Riccardo.Rattazzi@cern.ch
}

\begin{abstract}
I give a pedagogical introduction to the concepts and the tools that are necessary to study particle physics models in higher dimensions. I then give a more detailed presentation of warped compactifications and discuss their possible relevance to the hierarchy problem.
\end{abstract}

Keywords: Extra dimensions, branes, effective action, effective field theories, gravity, hierarchy, Kaluza-Klein fields, large extra dimensions, The RandallSundrum model, radions, moduli

\section{Introduction}

Science in general and particle physics in particular thrive from conceptual puzzles and unexplained phenomena. The gauge hierarchy problem is an exemplar source for inspiration. While we haven't got yet any direct experimental evidence onto what mechanism sets the small ratio $G_{N} / G_{F} \sim 10^{-34}$ between the Newton and Fermi constants, a great deal of theoretical progress in particle physics has been triggered in trying to come up with an explanation. For instance, the great development in supersymmetric field theory of the last three decades is to a good extent motivated by the potential relevance of supersymmetry to the hierarchy problem. The last few years have also witnessed a great revival in the interest for models with extra space dimensions. On one side this revival is motivated by important theoretical developments within superstring theory, in particular by the realization that there exist in string theory solitonic membranes, D-branes, on which ordinary particles could be localized [1]. On the other side the revival is also phenomenologically 
motivated by the realization that extra dimensions can shed a new light on the hierarchy problem [2].

The potential relevance of extra dimensions to the hierarchy problem can be grasped by the following simple line of reasoning. One way to phrase the hierarchy is that the Standard Model (SM) quanta, like the $Z$-boson, are much softer than the quanta of a possible underlying Grand Unified Theory (GUT) or string theory: $m_{Z} / m_{G U T} \sim$ $10^{-14}$. That is to say that the minimum frequency corresponding to a travelling $Z$ boson wave is $m_{Z} \sim 10^{2} \mathrm{GeV}$ while the minimal frequency of a GUT wave is $\sim 10^{16} \mathrm{GeV}$. Supersymmetry or technicolor allow for a dynamical explanation of this huge hierarchy. Moreover in both cases the value of $m_{Z} / m_{G U T}$ is determined by a quantum phenomenon, i.e. dimensional transmutation. However we know since long of a basic classical phenomenon that can make quanta softer: gravitational Redshift. Let us briefly recall how this works in general relativity. Consider a gravitational field specified by a metric $g_{\mu \nu}(x)$. The invariant interval separating event $x$ from event $x+d x$ is given by $(d s)^{2}=g_{\mu \nu} d x^{\mu} d x^{\nu}$. By the Equivalence Principle $(d s)^{2}$ equals the Lorentz invariant interval measured by any freely falling observer at $x$ : $(d s)^{2}=-\left(\Delta X_{0}\right)^{2}+\left(\Delta X_{1}\right)^{2}+\left(\Delta X_{2}\right)^{2}+\left(\Delta X_{3}\right)^{2}$. Consider the case of a static metric $g_{\mu \nu}$ and of an interval $d x$ in the time direction. In this case $(d s)^{2}=g_{00}\left(d x^{0}\right)^{2}<0$ corresponds to the proper time interval experienced by a freely falling observer with zero velocity at $x$

$$
d \tau=\sqrt{-g_{00}(x)} d x^{0}
$$

By the Equivalence Principle any clock at rest at $x$ will oscillate with its proper period $\Delta T=1 / \omega$ according to the same freely falling observer. Then by eq. (0.1) the frequency $\omega(x)$ oberved in the original reference frame will be rescaled according to

$$
\frac{1}{\omega}=\sqrt{-g_{00}(x)} \frac{1}{\omega(x)} .
$$

This rescaling is not yet, by itself, a physical effect: also the unit of measure of time at $x$, specified by some standard clock, will undergo the same rescaling. (Another way to state this is that the overall normalization of the metric, upon which eq. (0.2) crucially depends, is not an observable as it depends on the units of measure.) An observable effect arises when comparing the frequencies of two copies of the same clock located at different points. For instance we can consider two hydrogen atoms located respectively at points A and B in the gravitational field of a star (see Fig. (1)), and associate the frequency $\omega$ to a given atomic transition. The waves emitted at $\mathrm{A}$ will oscillate everywhere 


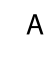

A

B

Figure 1. Two atoms $A$ and $B$ in the gravitational field of a star.

with frequency $\omega_{A}=\sqrt{-g_{00}(A)} \omega$. This is because the background is time independent, and two neighbouring wavecrests, leaving $\mathrm{A}$ with delay $1 / \omega_{A}$, will take the same time to reach any given point, for example point B. Similarly the wave emitted at B will oscillate everywhere with frequency $\omega_{B}=\sqrt{-g_{00}(B)} \omega$. The ratio

$$
\frac{\omega_{B}}{\omega_{A}}=\sqrt{\frac{g_{00}(B)}{g_{00}(A)}}<1
$$

represents a physical effect. The observer A notices that the light emitted from position B, closer to the star, is redshifted and similarly observer B notices that the light emitted at A is blue-shifted. This effect can be qualitatively understood as the photon loosing kinetic energy as it climbs up to A from deep inside the gravitational potential well at B. In everyday's life this gravitational redshift represents a tiny effect, since the gravitational field of the earth is rather weak. However gravity is a non-linear theory encompassing large gravitational fields, like the one near the horizon of a black hole. Assume indeed that the star of the previous example has collapsed to form a black hole. In this case the metric is given as a function of the radial coordinate $r$ by $g_{00}=-1+2 G_{N} M / r \equiv-1+r_{S} / r$ (with $M$ the black hole mass). As the position $r_{B}$ of atom B approaches the horizon $r_{S}$ we have $g_{00}\left(r_{B}\right) \rightarrow 0$ so that the the Redshift becomes infinite! The infinite Redshift of photons emitted at the horizon corresponds to the fact that light cannot escape from a black-hole. We can say that the gravitational field of a black hole creates an infinite hierarchy of energies of the emitted photons as the emitter is moved towards the horizon. With the gauge hierarchy in mind it is perhaps then natural to think of a wild generalization of the system we just discussed, one in which the points A or B are generalized to 3-dimensional spacelike surfaces, or 3-branes. In this process the dynamical system living on a point, the atom, is generalized to the dynamical system that lives on a 3 -surface, a $3+1$ quantum field theory, for 
instance the Standard Model. Imagine then to place two identical copies of 3-branes hosting the Standard Model at different points inside a gravitational field, in Fig. (2), in a straightforward generalization of Fig. (1). Of course, since three space dimensions already span the membranes, the distance separating them must correspond to a new spacelike dimension, the fifth dimension. In our generalization the role of the atomic energy levels (and thus of the emitted frequencies) is played by the masses of the particles living on the branes. For instance the masses of the two identical $Z$ bosons satisfy

$$
\frac{m_{Z}(B)}{m_{Z}(A)}=\sqrt{\frac{g_{00}(B)}{g_{00}(A)}}<1 .
$$

If only gravity propagates in the fifth dimension, one experimental consequence of eq. (0.4) is that the gravitons emitted in radiative $Z$ decays at point $\mathrm{A}$ are more energetic to observer $\mathrm{B}$ than those emitted in the same process at B. Also one can't stop from imagining a situation where brane B is much deeper than brane A inside a gravitational field, perhaps even very close to a horizon: then one would expect a huge hierarchy for the masses of identical particles living on the two different branes. Of course the example we are considering is not directly applicable to the gauge hierachy problem, as that does not concern two identical copies of the SM. Nevertheless the Redshifting mechanism would obviously be at work even if the field theories living on the two branes where not the same, and also in more complicated situations where the SM degrees of freedom are not fully localized: it is just kinematics. Is it then possible that the weak scale hierarchy originates as a consequence of gravitational Redshift in extra-dimensions? The answer to this question is affirmative and the model that proves it was proposed by Randall and Sundrum in a pioneering paper [3].

\section{Part I: Extra Dimensions}

In this section we introduce the players in the game (gravity, branes and localized fields) and discuss the rules that govern their effective action.

\subsection{Gravity and Branes}

Gravity plays a central role in the physics of extra dimensions. This is shown for instance by the example of the previous section. It is then important to recall the basic concepts relavant to describing the dynamics of gravity. A basic introduction to General Relativity is taken for granted here. We will be concerned with a D-dimensional space-time with co- 

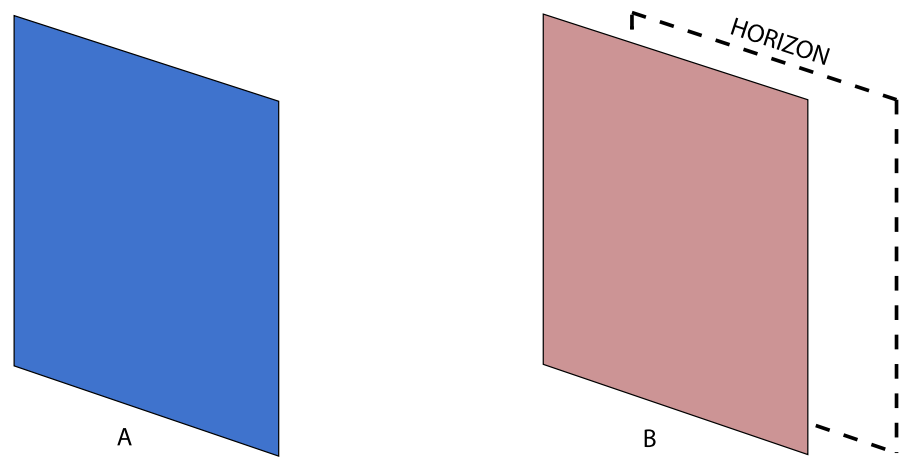

Figure 2. Two branes A and B in a 5-dimensional gravitational field.

ordinates $X^{M}$ and metric $g_{M N}(X)$, with $M, N=0, \ldots, D-1$. We use the mostly plus convention wherein at any space time point a locally inertial frame can be found in which $g_{M N}=\eta_{M N} \equiv(-1,+1, \ldots,+1)$. Later on we will specialize to the phenomenologically relevant case where $D-4$ space-like dimensions are compactified. The metric, up to diffeomorphisms, contains the dynamical degrees of freedom of gravity. The affine connection $\Gamma_{M N}^{R}=g^{R S}\left(\partial_{M} g_{N S}+\partial_{N} g_{M S}-\partial_{S} g_{M N}\right) / 2$ defines parallel transport, by means of which the Riemann tensor, characterizing the spacetime curvature, is constructed $\mathcal{R}_{M N R}^{S}=\partial_{N} \Gamma_{M R}^{S}-\partial_{M} \Gamma_{N R}^{S}+$ $\Gamma_{M R}^{T} \Gamma_{T N}^{S}-\Gamma_{N R}^{T} \Gamma_{T M}^{S}$. Using the Riemann tensor and its contracted forms, the Ricci tensor $\mathcal{R}_{M R}=\mathcal{R}_{M N R}^{N}$ and Ricci scalar $\mathcal{R}=\mathcal{R}_{M N} g^{M N}$, the most general invariant action can be written as

$S=\int d^{D} X \sqrt{g}\left\{a M_{D}^{D}+2 M_{D}^{D-2} \mathcal{R}+b M_{D}^{D-4} \mathcal{R}^{2}+\ldots c M_{D}^{D-6} \mathcal{R} \square \mathcal{R}+\ldots\right\}$

where $M_{D}$ is $D$-dimensional Planck scale. In ordinary 4-dimensional Einstein gravity, according to our non-conventional normalization, we have $M_{4}=\left(32 \pi G_{N}\right)^{-1 / 2} \simeq 1.2 \times 10^{18} \mathrm{GeV}$. We have parametrized all the couplings with dimensionless coefficients $a, b, c, \ldots$ and organized the lagrangian as a derivative expansion

$$
a M_{D}^{D} p^{0}+M_{D}^{D-2} p^{2}+b M_{D}^{D-4} p^{4}+\ldots+c M_{D}^{D-6} p^{6}+\ldots
$$

where the lowest terms are those that are important at the longest distance scales. In particular the first term, the cosmological constant, influences directly the global structure of the space-time. From the effective lagrangian point of view, which we will discuss in more detail later, we can meaningfully address only those phenomena that involve 
a finite number of terms, i.e. those for which $p / M_{D}$ is significatively less than 1 . In this respect, although one would naively expect all the coefficients $a, b, c, \ldots$ to be $O(1)$, we will assume that the cosmological term $a$ is much smaller than 1 . This is mostly for a theoretical reason: when $a=O(1)$ the background solution to Einstein equations has $\mathcal{R}=O\left(M_{D}^{2}\right)$ for which the derivative expansion breaks down. But notice also that there is a phenomenological preference to work with small cosmological constant. On one side we know from observation that the effective cosmological constant of our macroscopic 4-dimensional world is very small: $\Lambda^{4}=a_{4} M_{4}^{4} \sim\left(10^{-3} \mathrm{eV}\right)^{4} \ll M_{4}^{4}$. This may be an indication that also the fundamental cosmological constant $a M_{D}^{D-2}$, before compactification, is small. Moreover, as mentioned in sec. 1.1.6, a small $D$-dimensional cosmological constant is also favored in the scenario of large extra dimensions [2] by the requirement of a flat potential for the radius modulus. So, while we will assume $a \ll 1$ as a result of some tuning or, perhaps, $D$-dimensional supersymmetry, for all the other coefficients we will just need the perfectly natural and weak assumption that they be $\leq O(1)$. For instance in ordinary General Relativity with the above assumption the Einstein Lagrangian is a successful truncation up to the very small Planck length $\lambda_{P}=1 / M_{4}=10^{-33} \mathrm{~cm}$. This is evident with the above classical Lagrangian, but as discussed later, it remains true also at the quantum level.

The second important player in the extra dimensional game is given by the so called (mem)branes. They are extended objects which span surfaces and on which excitations (particles) can be localized. An explicit physical example of a brane is given, for instance, by the surface separating two different metals, where there exist localized excitations in the charge density, the surface plasmons. Another explicit example of a brane can be provided by a domain wall. Consider a scalar field theory with a $Z_{2}$ invariant potential $V(\phi)=\frac{g}{2}\left(\phi^{2}-v^{2}\right)^{2}$. In addition to the two vacuum solutions $\phi= \pm v$ this model contains domain wall solutions

$$
\phi=v \tanh \left(m\left(z-z_{0}\right)\right)
$$

where $m=\sqrt{g} v$ is the mass of the scalar field, while $z$ indicates one of the space directions. This solution interpolates between the $\phi=-v$ vacuum at $z=-\infty$ and the $\phi=+v$ vacumm at $z=+\infty$ and is thus topologically stable [4]. With respect to the true vacuum $\phi= \pm v$ this solution has an energy density $E=\left(\partial_{z} \phi\right)^{2} \propto \cosh ^{-4}\left(m\left(z-z_{0}\right)\right.$ localized within a distance $\sim 1 / m$ from the center of the wall. Integrating $E$ across the wall we obtain the wall tension $\tau=4 \sqrt{g} v^{3} / 3$. Away from the wall the scalar has mass $m$ so that at $E<m$ there are no modes 
propagating through the full space. There is however a massless scalar mode localized at the wall. Indeed the domain wall solution breaks spontaneously the original $D$-dimensional Poincaré group down to the $(D-1)$-dimensional one, corresponding to translations and boosts in the directions parallel to the wall. As it happens for ordinary internal symmetries, we then expect the presence of Goldstone bosons associated to the broken generators. A naive application of that result to spacetime symmetries is however not possible[7, 8]. The Goldstone theorem is proven by considering the local tranformations associated to the global symmetries. In the case of the Poincaré group both translations and boosts reduce, locally, to local translations. The Goldstone bosons are then in a one to one correspondence with the broken translation generators [7]. In the case at hand the translations along the $z$ direction are broken and $z_{0}$ parametrizes the manifold of equivalent vacua. Like in the case of internal symmetries we can parametrize the Goldstone excitation by promoting $z_{0}$ to a field $z_{0}(x)$ depending on the $(D-1)$ longitudinal coordinates. At linearized level it corresponds to a mode

$$
\delta \phi(x, z)=-\frac{v m}{\cosh ^{2} m z} z_{0}(x)
$$

which is clearly normalizable and localized within a distance $1 / m$ around the wall. Under $z$-translations $\phi(z, x) \rightarrow \phi(z+a, x)$ we have $z_{0}(x) \rightarrow$ $z_{0}(x)-a$. Because of this non linear symmetry the action can depend on $z_{0}$ only through its space time derivatives. In particular there is no mass term for $z_{0}(x)$. The effective action for $z_{0}$, valid at momentum $\ll m$, can be carefully derived by integrating out the massive excitations of the original field $\phi$. However it is intuitively clear what result to expect at lowest order. In this limit we are considering very smooth deformations of the wall, such that its position varies appreciably only over distances much bigger that its width $1 / \mathrm{m}$. At each point we then expect the field to be given approximately by eq. (1.3), but with $z$ replaced by the direction locally orthogonal to the wall. Then the action will just be given by the integral of the original wall tension $\tau=\sqrt{\lambda} v^{3}$ over the volume of the deformed wall.

Other fields can be localized at a domain wall. A fermion $\psi$ with Yukawa interaction $\lambda \psi \bar{\psi}$ wil be massive in the bulk, but will contain zero modes localized at the wall where $\phi=0$ [5]. Similarly, ideas to localized gauge fields have been proposed [6]. Likewise, D-branes in string theory support localized modes (scalars, fermions and vectors) associated to open strings ending on them [1]. In these lectures we will be focusing on the low energy description of branes. For instance, in the case we just considered this corresponds to $E \ll m$. In this regime we will not be concerned with the microscopic mechanism that gave origin 
to the brane and to the fields localized on it. We will just assume that the brane hosts a field theory of our choice and derive the consequences. The presence of some degrees of freedom, like the Goldstone $z_{0}$ above, could however just follow from symmetry considerations and not be an option.

\subsection{Brane Effective Actions}

We will now discuss the dynamics of branes by writing the most general effective action satisfying some basic principles. We follow closely the presentation given in ref. [7]. Let us consider an n-brane, a membrane filling $n$ spacial dimension whose spacetime trajectory, the worldbrane, is an $n+1$-surface. We parametrize this surface with coordinates $x^{\mu}$, $\mu=0, \ldots, n$. The embedding in the full $D$-dimensional spacetime is described by $D$ functions $X(x)^{M}$, with $M=0, \ldots, D-1$. For instance, in the simplest case of a point particle, a 0-brane, the worldbrane is the particle trajectory, the worldline, parametrized by a time coordinate $x^{0}$ : $X\left(x^{0}\right)$. It is physically intuitive that the distance between points on the brane, as measured by a brane observer, be the same as measured by a bulk observer,

$$
\begin{aligned}
\left.d s^{2}\right|_{\text {brane }} & =\left.\left.G_{M N}(X(x)) d X\right|_{\text {brane }} d X^{N}\right|_{\text {brane }} \\
& =G_{M N}(X(x)) \partial_{\mu} X \partial_{\nu} X^{N} d x^{\mu} d x^{\nu}
\end{aligned}
$$

i.e. the bulk metric gives rise to an induced metric $\hat{g}_{\mu \nu}$ on the brane

$$
\hat{g}_{\mu \nu}(x)=G_{M N}(X(x)) \partial_{\mu} X \partial_{\nu} X^{N} .
$$

Notice that the induced metric is a scalar under the bulk diffeomorphisms (all the $M, N \ldots$ indices are contracted) while it is a tensor under reparametrizations of the brane $x^{\mu}=x^{\mu}\left(x^{\prime}\right)$. As the choice of coordinates $x$ is arbitrary, physical quantities should not depend on it. Therefore, like in ordinary gravity, starting from $\hat{g}_{\mu \nu}$ we should write an action invariant under brane reparametrizations. We will do that in a moment. Before then we want to emphasize that, like we have projected the metric, so we can do with other tensors. For instance a bulk gauge field (1-form) $A_{M}(X)$ leads to a brane field $\hat{A}_{\mu}(x)=A_{M}(X(x)) \partial_{\mu} X^{M}$ which under the bulk gauge tranformation $A_{M} \rightarrow A_{M}+\partial_{M} \alpha$ shifts as under a proper $n+1$-dimensional gauge transformation

$$
\delta \hat{A}_{\mu}(x)=\partial_{M} \alpha(X(x)) \partial_{\mu} X^{M}(x)=\partial_{\mu}[\alpha(X(x))] .
$$

We can then use the projected field and gauge symmetry to couple the original 1-form to charged matter on the brane. A similar procedure 
can be followed for the D-bein field $E_{M}^{A}$, necessary to couple fermions to gravity in a manifestly covariant way. Here and in what follows we indicate with $A, B, \ldots, A=0, \ldots D-1$, and with $a, b \ldots, a=0, n$ respectively the bulk and brane Lorentz indices. The D-bein $E_{M}^{A}$ field represents $\mathrm{D} 1$-forms $(A=0, \ldots, D-1)$ in the cotangent space, satisfying the relation $\eta_{A B} E_{M}^{A} E_{N}^{B}=G_{M N}$. The D-bein defines at each space-time point a tangent space basis corresponding to the coordinates of a free falling observer: it associates to the entries of a vector $V^{M}$ in a given system of coordinates the entries $\hat{V}^{A}=E_{M}^{A} V^{M}$ in the coordinates of a free falling observer. Indeed, by the definition of $E_{M}^{A}$, vector products are conserved: $\hat{V}^{A} \hat{W}^{B} \eta_{A B}=V^{M} W^{N} G_{M N}$. Moreover local Lorentz rotations $E_{M}^{A}(X) \rightarrow R_{B}^{A}(X) E_{M}^{B}(X)$ are a gauge symmetry: the orientation of the $D$-bein at each point is not physical. This just means that the locally inertial reference frame is only defined up to a Lorentz transformation. Now, the tangent space $\sigma$ to the brane at a point $x$ is a $n+1$ subspace of the tangent space $\Sigma$ at $X(x)$. A vector $v^{\mu} \in \sigma$ is written in free falling coordinates as $\hat{v}^{A}=E_{M}^{A} \partial_{\mu} X^{M} v^{\mu}$. By this relation, $\sigma$ is represented as a $n+1$ dimensional subspace of the Lorentzian (free falling) vector space. To define the induced $n+1$-bein we have just to find an orthonormal basis of this subspace. One way to proceed is to divide the indices $\{A\}$ into two groups: $\{a\}$ for $A=0, \ldots, n$ and $\{i\}$ for $A=n+1, \ldots, D-1$. Since $\sigma$ is a time-like subspace we can always perform a Lorentz rotation $\hat{v}^{\prime A}=\bar{R}_{B}^{A} \hat{v}^{B}$ such that $\hat{v}^{\prime i} \equiv 0$ for $\hat{v}^{A} \in \sigma$. In the new basis, $\sigma$ is spanned by $\hat{v}^{\prime a}$ for $a=0, \ldots, n$, so that we have $\hat{v}^{\prime a} \hat{v}^{\prime b} \eta_{a b} \equiv \hat{v}^{A} \hat{v}^{\prime B} \eta_{A B}=v^{\mu} v^{\nu} g_{\mu \nu}$. For vectors in $\sigma$, summing over $a$ is equivalent to summing over $A$. The induced $n+1$-bein can then be defined as $e_{\mu}^{a} \equiv \bar{R}_{B}^{a} E_{M}^{B} \partial_{\mu} X^{M}$. It is straightforward to check that $e_{\mu}^{a}$ satisfies the basic conditions

$$
e_{\mu}^{a} e_{\nu}^{b} \eta_{a b}=\hat{g}_{\mu \nu} \quad e_{\mu}^{a} e_{\nu}^{b} \hat{g}^{\mu \nu}=\eta^{a b} .
$$

One crucial remark is that the rotation $\bar{R}_{B}^{A}$ is only defined modulo the rotations in the subgroup $S O(1, n) \times S O(D-n-1)$ which leave the $\sigma$ subspace and its complement invariant. In particular the induced $e_{\mu}^{a}$ is defined modulo the local Lorentz symmetry $S O(1, n)$ of the brane: the arbitrariness of our construction of $e_{\mu}^{a}$ does not affect physics provided the brane Lagrangian is written in a locally Lorentz invariant way. Using $e_{\mu}^{a}$ we can derive a brane spin connection and write a covariant Lagrangian for localized fermions.

The effective Lagrangian for a brane can then be written as

$$
\mathcal{S}_{\text {brane }}=\int d^{n+1} x \sqrt{g}\left\{-\tau+M^{n-1} \mathcal{R}(\hat{g})+\bar{\psi} \not D \psi+D_{\mu} \phi^{\dagger} D^{\mu} \phi+\right.
$$




$$
+ \text { (higher derivatives })\}
$$

where we have considered the example of localized fermion and scalar fields. The covariant derivatives involve the projected 1-forms, $\hat{A}_{\mu}$ and $e_{\mu}^{a}$, as well as any possible localized Yang Mills field. The most relevant term, the one with the lowest number of derivatives, corresponds to the brane tension $\tau$. In the effective theory description of the domain wall of the previous section, $\tau$ is determined by matching eq. (1.9) computed on a flat configuration, on which the intrinsic curvature terms vanish, with our calculation of the flat wall tension. This way we obtain $\tau=4 \sqrt{g} v^{3} / 3$. The general result in eq. (1.9) also shows that the naive derivation of the effective action sketched in sect. 1.1.1 is indeed accurate in the limit where the wall intrinsic curvature is small.

Let us consider as an explict example a 3-brane living in $D$ dimensional Minkowsky space, in the limit in which gravity is turned off. We can choose a gauge where the brane embedding is simply $X^{\mu}=x^{\mu}$ for $\mu=0,1,2,3$ and $X^{i}=Y^{i}(x)$ for $i=4, \ldots, D-1$. In this parametrization the brane roughly extends along the $0, \ldots, 3$ direction of the bulk space. The functions $Y^{i}$ parametrize the deformations along the orthogonal directions and are the dynamical degrees of freedom, the branons. In terms of the branons the induced metric is

$$
\hat{g}_{\mu \nu}=\eta_{\mu \nu}+\partial_{\mu} Y^{i} \partial_{\nu} Y^{j} \delta_{i j} .
$$

and the tension term of eq. (1.9) expanded in powers of $\partial Y$ becomes

$$
L_{e f f}=-\tau \sqrt{-} g=-\tau\left\{1+\frac{1}{2} \partial_{\mu} Y^{i} \partial^{\mu} Y_{i}+\frac{1}{8}\left(\partial_{\mu} Y^{i} \partial^{\mu} Y_{i}\right)^{2}+\ldots\right\} .
$$

This lagrangian provides a kinetic term with the right sign for $Y$ provided $\tau>0$. The configuration $Y^{i}=0$ is stable and represents the vacuum configuration of our brane. But the most remarkable thing is that our general symmetry considerations also fix all the interaction terms involving $n$ fields $Y$ and a number of derivatives $\leq n$. The terms involving the curvature affect the interactions that have always at least two more derivative, and give subleading contributions to the scattering of branons at low enough energy. This is totally analogous to what happens in ordinary sigma models, like the pion lagrangian of QCD, where at lowest order in the expansion $E / f_{\pi}$ the scattering amplitudes are fully fixed by the group structure in terms of just one physical parameter, $f_{\pi}$ itself. Indeed also the branon system is a $\sigma$-model whose coset space corresponds to the breakdown of the $D$-dimensional Poincaré group down to the 4 -dimensional one $[7,8]$. To conclude, notice that by indicating 
$\tau=f^{4}$ and by going to the canonical field $\hat{Y}^{i}=f^{2} Y^{i}$, the branon interactions are proportional to inverse powers of $f$ : this mass scale plays a role analogous to $f_{\pi}$ in the pion Lagrangian.

\subsection{Effective Field Theories}

One general aspect of physical systems is that the dynamics at large length scales, or equivalently at low energy, does not depend too much on the microscopic details. For instance the interaction of an electromagnetic wave with an antenna of size $a$ much smaller than the wavelength $\lambda$ is described to a good accuracy by the coupling to the dipole mode of the antenna. Higher multipole moments will contribute to corrections suppressed by powers of $\lambda a \ll 1$. Another example is provided by molecules, where the slow vibrational modes, describing the oscillations in the distance between the various nuclei, can be accurately studied by first averaging over the fast motions of electrons. Averaging over the electronic states provides an effective Hamiltonian for the low frequency modes, where the higher details of the electronic structure are controlled by higher powers of the ratio $\omega_{\text {slow }} / \omega_{\text {fast }}$. Effective Field Theories technique provide a systematic way, an expansion, to treat the details of microscopic physics when discussing phenomena at low enough energy. Normally, when Quantum Field Theory is introduced as a construction to describe fundamental processes, a great emphasis is put on the requirement of renormalizability. Technically renormalizability corresponds to the possibility of sending the energy cut-off $\Lambda$ of the system to infinity while keeping all the physical quantities finite (and non trivial)*. Physically this means that the theory can be extrapolated to infinitely small distances without encountering new microscopic structures. Renormalizable theories can be truly fundamental and not just an effective description valid in a limited energy range. Renormalizable theories are however a special case, and in practically all applications to particle physics one deals with non-renormalizable effective field theories $\dagger$ The best example of a non-renormalizable theory is given by General Relativity, which necessarily requires a new description at an energy scale smaller or equal to $M_{P}=10^{19} \mathrm{GeV}$. Nonetheless GR makes perfect sense as an effective field theory at energies much smaller than its cut off [10]. But also QED is an effective field theory: the interactions of electrons and photons are modified at energies much bigger than $m_{e}$ by

\footnotetext{
* As we will explain better below this is a somewhat stronger requirement than renormalizability: in weakly coupled theories it corresponds to asymptotic freedom.

$\dagger$ For excellent introductions to effective field theories see the papers in Ref.[9]
} 
the presence of new particles and new interactions. Still in the regime $E \sim m_{e}$ the small effects of the microscopic dynamics can be accounted for by adding a suitable tower of non-renormalizable interactions. The $\mathrm{SM}$, the most fundamental description of particle interactions gravity excluded, is renormalizable. Still we can only consider the SM as an effective theory. On one hand this is because the necessary inclusion of gravity makes it non-renormalizable. On the other hand, even in the absence gravity, the SM is renormalizable but not asymptotically free. At least one of its couplings, the one associated to the hypercharge vector boson, grows logarithmically with energy and becomes infinite at a scale $M_{L} \sim m_{W} e^{b / \alpha}$, with $b=12 \pi \cos ^{2} \theta_{W} / 41 \sim 1$. At $E \sim M_{L}$ the perturbative description breaks down, very much like the effective description of G.R. breaks down at the Planck scale. The fact that $M_{L} \gg M_{P}$ makes however this second problem academic.

In order to make these general statements more concrete, let us focus on a very simple example. Let us consider a physical system which at low enough energy possesses just one scalar degree of freedom parametrized by a field $\phi$. The most general local and Poincaré invariant Lagrangian can be written as an expansion in powers of $\phi$ and of its derivatives

$$
\begin{aligned}
\mathcal{L} & =\partial_{\mu} \phi \partial^{\mu} \phi-m^{2} \phi^{2}+\lambda_{4} \phi^{4}+\frac{\lambda_{6}}{M^{2}} \phi^{6}+\frac{\lambda_{8}}{M^{4}} \phi^{8}+\cdots \\
& +\frac{\eta_{4}}{M^{2}} \phi^{2} \partial_{\mu} \phi \partial^{\mu} \phi+\frac{\eta_{6}}{M^{4}} \phi^{4} \partial_{\mu} \phi \partial^{\mu} \phi \cdots
\end{aligned}
$$

where for simplicity we have also assumed a symmetry $\phi \rightarrow-\phi$. We have scaled all the couplings by powers of one mass scale $M$ and by dimensionless quantities $\lambda_{i}, \eta_{i}, \ldots$. It is reasonable to assume that $\lambda_{i}, \eta_{i}, \ldots \sim$ $O(1)$. This corresponds to a theory that in addition to the particle mass $m$ contains only another physical scale, $M$, associated to the interactions. It is easy to understand the meaning of this expansion when calculating scattering amplitudes at energies $m \ll E \ll M$. Let us focus on tree level computations first. We shall worry about quantum corrections later. Neglecting numerical factors and indicating one power of momentum generically by $E$, we have

$$
\mathcal{A}_{2 \rightarrow 2}(E) \sim \lambda_{4}+\eta_{4} \frac{E^{2}}{M^{2}} \ldots
$$

for the elastic process $2 \rightarrow 2$ corresponding to Fig. (3) and

$$
\mathcal{A}_{2 \rightarrow 4}(E) \sim \frac{1}{E^{2}}\left\{\lambda_{4}^{2}+\lambda_{4} \eta_{4} \frac{E^{2}}{M^{2}}+\lambda_{6} \frac{E^{2}}{M^{2}}+\ldots\right\}
$$

for the inelastic process $2 \rightarrow 4$ shown in Fig. (4). This power counting corresponds to simple dimensional analysis. Notice that for $E \ll M$ 


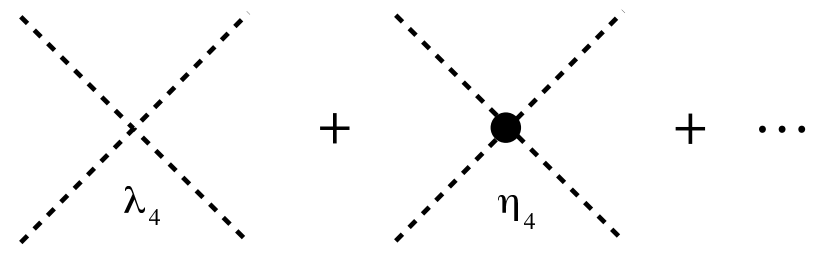

Figure 3. The diagrams contributing to the elastic process $2 \rightarrow 2$ at lowest order.

the dimensionless coupling $\lambda_{4}$ dominates all the amplitudes. This result is intuitively obvious. A coupling $g$ of mass dimension $[E]^{d}$ can perturbatively contribute to observables via the dimensionless combination $g / E^{d}$. We can then distinguish tree classes of couplings depending on whether $d$ is positive, zero or negative. Couplings of positive dimension are called relevant, as their effect becomes more important the smaller the energy. An example is given by the mass term itself, which gives small $O\left(\mathrm{~m}^{2} / E^{2}\right)$ effects in the relativistic regime, but becomes important when $E \sim O(m)$. Couplings of vanishing dimensions, like $\lambda_{4}$, are termed marginal. At tree level their effects are independent of the energy scale. Finally, couplings of negative dimension are termed irrelevant, as their effects become very small in the low energy domain. Notice that while there is only a finite numer of relevant and marginal couplings, the tower of irrelevant couplings is infinite. In spite of their infinity, and as their naming suggests, irrelevant couplings do not totally eliminate the predictive power of our Lagrangian as long as we use it at low energy, $E \ll M$. At each finite order $(E / M)^{n}$, only a finite number of terms in the Lagrangian contributes to the amplitudes. This preserves a weak form of predictivity, which is often good enough, since we just need to match our theoretical computations to the experimental precision, which is always finite.

We can now worry about quantum corrections. These introduce some technical difficulties, but the basic conclusion is unchanged. To be fully general, let us write our Lagrangian as a sum over operators $\mathcal{O}_{i}$ of dimension $d_{i}+4$

$$
\mathcal{L}=\sum_{i} c_{i} \frac{\mathcal{O}_{i}}{M^{d_{i}}}
$$

Assume we want to calculate some observable at order $(E / M)^{n}$. Working at tree level it is enough to truncate $\mathcal{L}$ to the operators with $d_{i} \leq n$. The analysis at tree level is made simple by the fact that the external momenta $(\sim E)$ completely fix the momenta of the internal lines and vertices. This is no longer true at loop level, where the loop mo- 


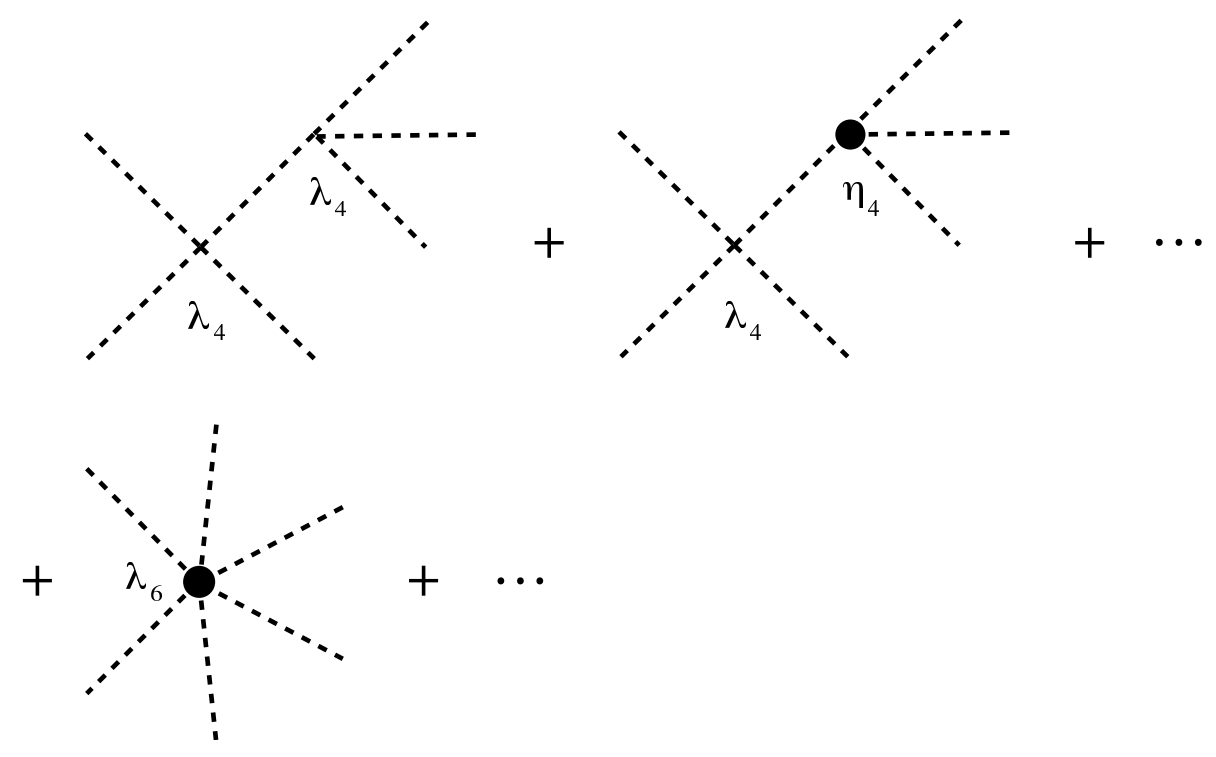

Figure 4. The leading contributions to the inelastic process $2 \rightarrow 4$.

mentum can be arbitrarily high. Moreover some of the loop integrals are UV divergent and must be cut-off at some scale $\Lambda$. An interaction term $c \mathcal{O}_{N} / M^{d_{N}}$, can generate quantum corrections that involve positive powers of the cut-off $\Lambda$

$$
\frac{\delta \mathcal{A}}{\mathcal{A}} \sim \cdots+c \frac{\Lambda^{P} E^{d_{N}-P}}{M^{d_{N}}}+\cdots
$$

Then an operator with $d_{N}>n$, which at tree level only gives corrections beyond the needed accuracy $E^{n}$, can, at loop level, generate effects that scale with a power $d_{N}-P \leq n$. Moreover if $\Lambda \sim O(M)$ these quantum effects are as important as the tree level contribution of operators of lower dimension. This seems very embarrassing. Fortunately it can be proven that these effects are exactly equivalent to a renormalizations of the coefficients $c_{i}$ of the operators of lower dimensionality. Therefore they do not contain any new information and can be eliminated by a trivial change of renormalization scheme. Their equivalence to local operators is qualitatively understandable: loops of high virtuality are small in position space, corresponding to a region of size $1 / \Lambda$, and look pointlike with respect to the long wavelength $1 / E$ of the external particles. Another way to understand this result is to take a Wilsonian view point where $\Lambda$ is the running cut-off. After running down to a scale $\Lambda$ such 


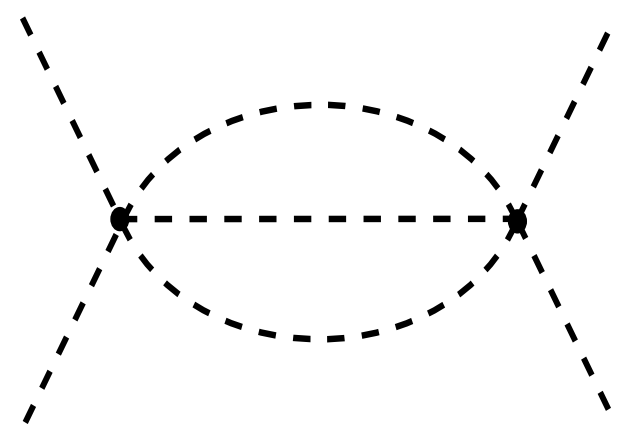

Figure 5. 2-loop contribution to elastic scattering from $\lambda_{5} \phi^{5} / M$ vertices.

that $E \lesssim \Lambda \ll M$, the troublesome virtual effects becomes manifestly small: the big effect has been replaced by a local renormalization of the classical Lagrangian ${ }^{\ddagger}$. But there is no doubt that the most convenient method to define effective field theories at the quantum level is by Dimensional Regularization (DR). Dimensionally regulated loop integrals exhibit no powerlike divergences, only logarithmic divergences survive. The issue we just worried about does not even arise! The naive power counting we found at tree level carries over to the quantum theory up to mild logarithimic corrections.

Consider, for instance, the 2-loop diagram involving two insertions of the $\lambda_{5} \phi^{5} / M$ interaction shown in Fig. (5) By using a hard momentum cut off we have

$$
\delta \mathcal{A}_{2 \rightarrow 2}=\lambda_{5}^{2}\left\{a \frac{\Lambda^{2}}{M^{2}}+b \frac{E^{2}}{M^{2}} \ln \Lambda / E\right\}
$$

while DR in $4+\epsilon$ dimensions gives

$$
\delta \mathcal{A}_{2 \rightarrow 2}=\lambda_{5}^{2}\left(\frac{1}{\epsilon}+b \ln \mu / E\right) \frac{E^{2}}{M^{2}} .
$$

In DR, after renormalization, this diagram gives just a logarithimic Renormalization Group (RG) evolution of the coefficient of a dimension 6 operator $\left(\phi^{2}\right) \square\left(\phi^{2}\right)$. We emphasize that while the power divergences are totally scheme dependent, being fully saturated in the UV, the logarithmic divergence involves a physical IR singularity $\ln E$ and

\footnotetext{
¥T make this argument fully rigorous one should take into account that the Wilsonian Lagrangian at scale $\Lambda$ now also contains terms that scale like inverse powers of $\Lambda$. Terms proportional to $1 /\left(\Lambda^{R} M^{S}\right)$ are however fixed by the renormalized couplings associated to operators with $d_{i} \leq S[11]$.
} 
must be the same in both regularizations. This $\ln E$ term is associated by unitarity to the cut diagrams $\left|\mathcal{A}_{2 \rightarrow 3}\right|^{2}$.

A by-product of this discussion is that in DR with minimal subtraction (or any other mass independent subraction scheme) the RG equation for the couplings of an effective Lagrangian follows just by dimensional analysis [12]. Using the notation of eq. (1.15) where a coupling $c_{i} / M^{d_{i}}$ has dimension $-d_{i}$, the $\beta$ function has the form

$$
\mu \frac{d c_{i}}{d \mu}=\sum_{d_{j}+d_{k}=d_{i}} a_{j, k} c_{j} c_{k}+\sum_{d_{j}+d_{k}+d_{l}=d_{i}} a_{j, k, l} c_{j} c_{k} c_{l}+\cdots
$$

where $a_{i, j}, a_{i, k, l}, \ldots$ are numerical coefficient following from the loop integrals. Notice that the parameter submanifold where all the irrelevant couplings $\left(-d_{i}<0\right)$ vanish is stable under RG evolution. This interesting submanifold corresponds to what we normally call renormalizable theories. On the other hand, once we turn on an irrelevant coupling of dimension $-d<0$ it will generate by RG evolution an infinite subset of the couplings of more negative dimension. Such theories are termed non-renormalizable, as quantum effects force the presence of infinitely many inputs, though we hope to have made it clear how to deal with them. Notice also that our original assumption to scale all the irrelevant couplings by the same mass $M$ is stable under RG flow. Of course there can be more complicated situations and models where the higher dimensional couplings involve hierarchically different scales.

We conclude this discussion by reiterating the basic theorem. Lagrangians involving all possible non-renormalizable terms can be made sense of as effective ones. A weak form of predictivity can be preserved by working in perturbation theory in an expansion in $E / M$, where $M$ is the lowest scale characterizing the non-renormalizable couplings. This works as long as $E \ll M$. When $E \sim M$ infinitely many parameters become relevant and our effective Lagrangian completely looses predictive power. A reasonable expectation is that at the scale $M$ the theory enters a new regime where perhaps new degrees of freedom are relevant. For instance this is what happens in QCD at the scale $4 \pi f_{\pi} \sim 1 G e V$ where the weakly coupled description of mesonic physics breaks down. At this energy the hadrons deconfine and at higher energies the dynamics is more accurately described in terms of quarks and gluons. Notice that, according to our discussion, non-asymptotically free renormalizable theories are qualitatively similar to non-renormalizable theories. In the former case, at least one coupling grows logarithmically $\lambda(E) \propto 1 / \ln \left(M_{L} / E\right)$ with energy, while in the latter the growth of the effective dimensionless couplings is powerlike $\lambda(E) \propto(E / M)^{n}$. In both cases the perturbative description breaks down at some high scale, $M_{L}$ or $M$. The only differ- 
ence between the two cases is quantitative: in the renormalizable case, for a not too small a value of the running coupling at low energy, the cut-off scale $M_{L}$ is exponentially far away.

\subsection{Examples}

We can analyze from the effective field theory viewpoint some system of interest. One instructive example is provided by pure gravity, whose Lagrangian was given in eq. (1.1). To study the interactions let us focus on the case of vanishing cosmological constant and let us expand the metric field around the flat background

$$
g_{M N}=\eta_{M N}+\frac{h_{M N}}{M_{D}^{\frac{D}{2}-1}} .
$$

Eq. (1.1) will then be written as a power series in the fluctuation $h$

$$
\begin{array}{r}
\left\{(\partial h)^{2}+\frac{1}{M_{D}^{\frac{D}{2}-1}} h(\partial h)^{2}+\frac{1}{M_{D}^{D-2}} h^{2}(\partial h)^{2}+\ldots\right\} \ldots \\
+b\left\{\frac{1}{M_{D}^{\frac{D}{2}+1}}\left(\partial^{2} h\right)\left(\partial^{2} h\right)+\ldots\right\}+\ldots
\end{array}
$$

where we have been very schematic, suppressing all the tensor structure indices and $O(1)$ factors, but keeping the derivative expansion structure manifest. Notice that the fluctuation $h$ has been defined in such a way that it is canonically normalized. The interactions then all scale by inverse powers of the Planck mass. The above Lagrangian, after suitable gauge fixing can be used to compute graviton scattering processes in perturbation theory. For instance the amplitude $\mathcal{A}_{2 \rightarrow 2}$ scales with the energy $E$ like

$$
\mathcal{A}_{2 \rightarrow 2} \sim \frac{E^{D-2}}{M_{D}^{D-2}}\left(1+b \frac{E^{2}}{M_{D}^{2}}+\ldots\right) .
$$

The dots also include quantum effects, which scale like positive powers of $E / M_{D}$. For instance, by simple dimensional analysis, 1-loop effects induced by the leading two derivative Lagrangian are of order $\left(E / M_{D}\right)^{D-2}$ with respect to the leading tree level contribution. In the ordinary purely 4 dimensional theory of Einstein gravity the Planck mass $M_{4} \sim 10^{18}$ $\mathrm{GeV}$ is much bigger than any energy scale relevant to astrophysics or cosmology (if not for very early cosmology, even before inflation). Then the truncation of the theory to the lowest two derivative Lagrangian, the Einstein-Hilbert action, already allows a very accurate description of the dynamics. 
As a second example consider the interactions of the branon excitations $Y$ of a 3-brane

$$
\mathcal{L}_{\text {brane }}=-f^{4} \sqrt{\hat{g}}+b f^{2} \sqrt{\hat{g}} \mathcal{R}(\hat{g})+\ldots
$$

Substituting eq. (1.10) and writing the interactions in terms of the canonical fields $\hat{Y}_{i}=f^{2} Y_{i}$ it is straighforward to power count the scaling of Feynman diagrams. For the $Y Y \rightarrow Y Y$ amplitude the Feynman diagram expansion corresponds to the series

$$
\mathcal{A}_{2 \rightarrow 2}=\frac{E^{4}}{f^{4}}\left(1+C \frac{E^{4}}{f^{4}} \ln E+b \frac{E^{2}}{f^{2}}+\ldots\right) .
$$

where the first and second term (proportional to a calculable coefficient $C$ ) are determined by the quartic interaction in eq. (1.11) respectively at tree level and at 1-loop. The tension $f$ turns out to be the energy scale which controls the perturbative expansion. At energies $E \sim f$ the effective field theory description surely breaks down, in analogy with the case $E \sim M$ in the scalar toy model of the previous section. The quantity $(E / f)^{2}$ controls the strength of the interaction like $\alpha / 4 \pi$ does in quantum electrodynamics.

The length $L=1 / f$ can be interpreted as the quantum size of the brane, in analogy with the Compton wavelength of a particle. Indeed in the case of a 0-brane, a point particle, $f$ coincides with the mass $m$ and we recover the usual definition of Compton wavelength. In the case of a particle the length $1 / \mathrm{m}$ controls the domain of validity of the low energy non-relativistic effective theory. If we try to localize one electron at a distance $<1 / m$, then, by the indetermination principle, not only will its momentum $p$ be relativistic but the production of electron positron pairs energetically possible. In the case of the brane, we can, for instance, consider the quantum fluctuation of the linearized induced metric on the vacuum . We find (cfr. eq. (1.10))

$$
\left\langle\partial_{\mu} Y^{i} \partial^{\mu} Y_{i}\right\rangle \sim \frac{1}{f^{4}} \int k^{3} d k
$$

which shows that at wavelengths of order $1 / f$ the fluctuation of the brane position becomes itself of order $1 / f$ : at these short wavelengths the brane cannot be approximated by a smooth surface. Basically it is not possible to talk about fluctuations in the position of the brane that are shorter than $1 / f$ in both longitudinal and transverse directions.

With the previous considerations in mind, it is instructive to consider the field theoretic domain wall discussed in sect. 1.1.1. For definiteness let us focus on the case of a 4-dimensional scalar theory, so that the wall 


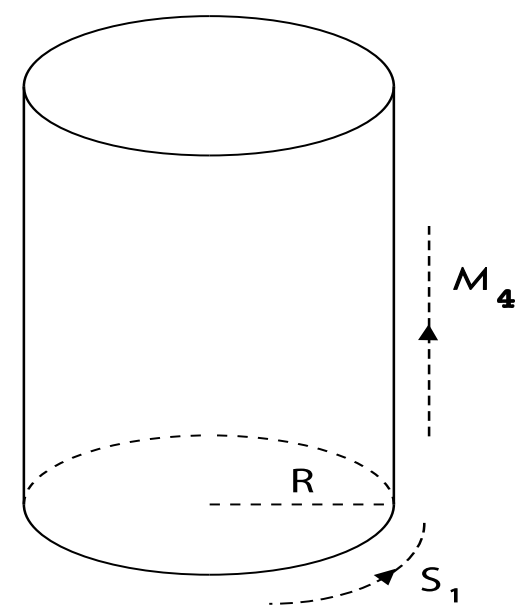

Figure 6. Cylindrical structure of 5-dimensional space-time compactified on $\mathcal{M}_{4} \times$ $S_{1}$.

is a 2-brane. The tension is $\tau=\sqrt{g} v^{3}$, while the cut-off of the effective description is provided by $m=\sqrt{g} v$, the energy at which extra massive modes come in. We have $m=(g \tau)^{1 / 3}$, so that as long as the original 4 D theory was weakly coupled $(g \ll 1)$, the brane theory never gets into a strong coupling regime. At the cut off scale $m$, the loop expansion parameter of the effective brane theory $m^{3} / \tau=g$ coincides with the loop expansion parameter of the original scalar field theory.

\subsection{Kaluza-Klein decomposition}

So far we have been general: our discussion applies equally well to compact and to infinite extra dimensions. However, since it is empirically very clear that we live in three macroscopic spatial dimensions, for phenomenological applications we must focus on the case in which the extra-dimensions are compactified at some small enough radius $R$. The dynamics at distances much bigger than $R$ will not be able to notice the presence of the extra compact directions. To illustrate this fact let us consider the simplest situation of a 5D scalar field $\phi$ with the 5th dimension compactified on a circle (see Fig. (6)) of radius $R$. Compactification is formally expressed by the periodicity requirement

$$
\phi\left(x, x_{5}\right)=\phi\left(x, x_{5}+2 \pi R\right)
$$

Processes taking place on time scales $T \ll R$, by causality and by locality, cannot notice that the 5th dimension is compact. On the other hand 
to study processes happening on a time scale $T \gtrsim R$, and in particular at energies $E \lesssim 1 / R$, the $5 \mathrm{D}$ local description is not the most adequate. In this case it is convenient to expand the field $\phi$ in its Fourier components with respect to the periodic coordinate $x_{5}$.

$$
\phi\left(x, x_{5}\right)=\sum_{n=-\infty}^{n=\infty} \phi_{n}(x) e^{i \frac{n x_{5}}{R}} .
$$

where the reality of $\phi$ implies $\phi_{-n}(x)=\phi_{n}(x)^{*}$. Notice that each different coefficient $\phi_{n}$ in this expansion corresponds to a different $4 \mathrm{D}$ field. The $\phi_{n}$ are called Kaluza-Klein (KK) fields. According to this expansion the $5 \mathrm{D}$ kinetic action integrated over $x_{5}$ becomes

$$
\begin{aligned}
& \int \mathcal{L}_{\phi} d x^{5}=-\frac{1}{2} \quad \int\left[\left(\partial_{\mu} \phi\right)^{2}-\left(\partial_{5} \phi\right)^{2}\right]= \\
& \frac{1}{2} \quad \sum_{-\infty}^{\infty}\left[-\left|\partial_{\mu} \phi_{n}\right|^{2}+\frac{n^{2}}{R^{2}}\left|\phi_{n}\right|^{2}\right] \text {. }
\end{aligned}
$$

The original 5D massless field has been decomposed in a tower of KaluzaKlein scalars $\phi_{n}$ with mass

$$
m_{n}=n / R .
$$

If we work at energy $E$, only a limited number $n \sim E R$ of KK can be produced. In particular, for $E<1 / R$ only the zero mode $\phi_{0}$ is available. At such low energies the model looks 4-dimensional. The KK particles appear only virtually, and their effect is reproduced by a suitable set of local operators involving only the massless $4 \mathrm{D}$ fields. In the specific example we are considering, the full space-time symmetry is just the 4dimensional Poincaré group times translations along the fifth direction: $P_{4} \times U(1)$. The KK particle states represent just the irreducible representations of this group. In particular the index $n$ represents the charge under the $U(1)$ group of $5 \mathrm{D}$ translations: $5 \mathrm{D}$ translational invariance shows up in $4 \mathrm{D}$ as the conservation of the KK indices $n_{i}$ summed over the incoming and outgoing particles in a collision.

Along similar lines one can study the KK decomposition of a gauge vector field $A_{M}$. But rather than discussing it in detail we go directly to the case of the graviton: the technical issues, associated to gauge invariance, are analogous for both vector and tensor field. So let us consider the original theory of Kaluza and Klein [13]: 5D Einstein gravity compactified on $\mathcal{M}_{4} \times S_{1}$ with the action

$$
2 M_{5}^{3} \int_{\mathcal{M}_{4} \times S_{1}} \sqrt{g} \mathcal{R}(g)
$$


We can write the full metric tensor in block form

$$
g_{M N}\left(x, x^{5}\right)=\left(\begin{array}{ll}
g_{\mu \nu} & g_{\mu 5} \\
g_{5 \mu} & g_{55}
\end{array}\right)=\left(\begin{array}{cc}
\eta_{\mu \nu}+h_{\mu \nu} & h_{\mu 5} \\
h_{\mu 5} & 1+h_{55}
\end{array}\right) .
$$

To work out the spectrum we must compute the quadratic action in the linearized field $h_{M N}$ and then use the gauge freedom provided by the linearized 5D diffeomorphisms, $x^{M} \rightarrow x^{M}+\epsilon^{M}\left(x, x^{5}\right)$

$$
h_{M N} \rightarrow h_{M N}+\delta h_{M N}=h_{M N}+\partial_{N} \epsilon_{M}+\partial_{M} \epsilon_{N} .
$$

to eliminate the redundant degrees of freedom. Here and in what follows, working at linear order, indices are raised and lowered using the Lorentz metric $\eta_{M N}$. We stress that the compactification of the fifth dimension implies that all our fields, including $\epsilon_{M}$ are periodic in $x^{5}$. Using the 5 gauge parameters $\epsilon_{N}$ we can essentially eliminate 5 combinations of the metric fluctuations $h_{M N}$. We can choose these 5 combinations to be just $h_{55}$ and $h_{\mu 5}$. By using Fourier modes we have that $\delta h_{55}=2 \partial_{5} \epsilon_{5}$ becomes

$$
\delta h_{55}^{(n)}=2 i n \epsilon_{5}^{(n)}
$$

which explicitly shows that we can eliminate all the modes but $h_{55}^{(0)}$, which is gauge invariant. The gauge invariance of zero modes like $h_{55}^{(0)}$ follows from the periodicity of the gauge transformation

$$
\oint_{S_{1}} \delta h_{55}=2 \oint_{S_{1}} \partial_{5} \epsilon_{5}=0
$$

and is a generic features of gauge theories on compact spaces. The same thing happens for $h_{\mu 5}$

$$
\delta h_{\mu 5}^{(n)}=\partial_{\mu} \epsilon_{5}^{(n)}+i n \epsilon_{\mu}^{(n)} .
$$

Therefore by using the $n \neq 0$ modes of $\epsilon_{M}$ we can go to a gauge where

$$
h_{55}\left(x, x_{5}\right) \equiv \phi(x) \quad h_{\mu 5}\left(x, x_{5}\right) \equiv A_{\mu}(x)
$$

while $h_{\mu \nu}\left(x, x_{5}\right)$ is unconstrained. However we still have the zero mode gauge freedom

$$
A_{\mu} \rightarrow A_{\mu}+\partial_{\mu} \epsilon^{(0)} \quad h_{\mu \nu}^{(0)} \rightarrow h_{\mu \nu}^{(0)}-\partial_{\mu} \epsilon_{\nu}^{(0)}-\partial_{\nu} \epsilon_{\mu}^{(0)} .
$$

The residual scalar mode $\phi$, usually called radion, is associated to fluctutations in the proper length $L$ of the radius of compactification $\delta L=$ $\oint h_{55} / 2=\pi R \phi$. The graviphoton $A_{\mu}$, as shown in eq. (1.37), is the gauge field associated to $4 \mathrm{D}$ local translations of the 5 th coordinate. 
The associated charge is just the momentum along the fith dimension, i.e. the KK index $n$.

Defining the KK modes via

$$
h_{\mu \nu} \equiv \sum_{n=-\infty}^{+\infty} h_{\mu \nu}^{(n)} e^{i \frac{n x_{5}}{R}}
$$

the linearized $4 \mathrm{D}$ action becomes

$$
\begin{aligned}
\mathcal{L}_{4 D}^{(2)}=M_{5}^{3} \pi R & \left\{\left[\sum_{-\infty}^{+\infty} h^{(n)^{\mu \nu}} \square h_{\mu \nu}^{(-n)}-h_{\mu}^{(n)}{ }_{\mu}^{\mu} \square h^{(-n)^{\nu}}\right.\right. \\
& +2 h_{\mu \nu}^{(n)} \partial^{\mu} \partial^{\nu} h^{(-n)^{\rho}}{ }_{\rho}-2 h_{\mu \nu}^{(n)} \partial^{\mu} \partial^{\rho} h^{(-n)^{\nu}} \\
& \left.+\quad \frac{n^{2}}{4 R^{2}}\left(h^{(n)}{ }_{\mu}^{\mu} h^{(-n)^{\nu}}-h^{(n)}{ }^{\mu \nu} h_{\mu \nu}^{(-n)}\right)\right] \\
& \left.+2 \phi\left(\partial^{\mu} \partial^{\nu} h_{\mu \nu}^{(0)}-\square h_{\mu}^{(0)}{ }_{\mu}^{\mu}\right)-F_{\mu \nu} F^{\mu \nu}\right\}
\end{aligned}
$$

where $F_{\mu \nu}=\partial_{\mu} A_{\nu}-\partial_{\nu} A_{\mu}$. By looking at the coefficient of the zero mode action we deduce that the effective 4-dimensional Planck scale is

$$
M_{4}^{2} \equiv M_{5}^{3} 2 \pi R
$$

The $\partial_{5}$ terms of the $5 \mathrm{~d}$ Lagrangian have turned into mass terms for the $n \neq 0$ modes. As first noticed by Fierz and Pauli [14], the specific tensor structure of this mass term is the only one ensuring the absence of ghosts and tachyons in $h_{\mu \nu}^{(n)}$. The equations of motion for the massive modes reduce indeed to

$$
\left(\square+\frac{n^{2}}{R^{2}}\right) h_{\mu \nu}^{(n)}=0 \quad \partial^{\mu} h_{\mu \nu}^{(n)}=h_{\mu}^{(n)^{\mu}}=0
$$

where the second and third relations follow by taking the divergence and trace of the equation of motion. This is completely analogous to the well known case of a massive vector $V_{\mu}$. There the divergence of the equation of motion gives the constraint $\partial^{\mu} V_{\mu}=0$, implying that only 3 out of the 4 degrees of freedom propagate, as it should be for a $J=1$ massive particle. Here, due to the constraints, we have $10-$ $5=5$ propagating states, corresponding to a massive $J=2$ particle. An arbitrary symmetric two index tensor $H_{\mu \nu}$ can be decomposed in components of definite spin as

$H_{\mu \nu}=H_{\mu \nu}^{T T}+\partial_{\mu} E_{\nu}^{T}+\partial_{\nu} E_{\mu}^{T}+\left(\eta_{\mu \nu}-\frac{\partial_{\mu} \partial_{\nu}}{\partial^{2}}\right) \Phi+\frac{\partial_{\mu} \partial_{\nu}}{\partial^{2}} \Psi \equiv 2 \oplus 1 \oplus 0_{\Phi} \oplus 0_{\Psi}$ 
where $E_{\mu}^{T}$ and $H_{\mu \nu}^{T T}$ are respectively transverse and transverse-traceless $\left(\partial^{\mu} E_{\mu}^{T}=\partial^{\mu} H_{\mu \nu}^{T T}=\eta^{\mu \nu} H_{\mu \nu}^{T T}=0\right)$ and the spin of each component is indicated in an obvious notation. By eq. (1.41) only $H^{T T}$ survives on-shell. An instructive exercise is to construct the projectors on $H^{T T}, E^{T}, \Phi$ and $\Psi$ by writing them in a compact way in terms of the transverse and longitudinal vector projectors $\Pi_{\mu \nu}^{T}=\eta_{\mu \nu}-\partial_{\mu} \partial_{\nu} / \partial^{2}, \Pi_{\mu \nu}^{L}=\partial_{\mu} \partial_{\nu} / \partial^{2}$. Another instructive exercise is to write the kinetic Lagrangian in terms of the various projectors, in complete analogy with the massive $J=1$ case, and from that to derive the massive $J=2$ propagator

$$
\left\langle h_{\mu \nu}^{(-n)} h_{\rho \sigma}^{(n)}\right\rangle=\frac{\frac{1}{2}\left(\hat{\Pi}_{\mu \rho}^{T} \hat{\Pi}_{\nu \sigma}^{T}+\hat{\Pi}_{\mu \sigma}^{T} \hat{\Pi}_{\nu \rho}^{T}\right)-\frac{1}{3} \hat{\Pi}_{\mu \nu}^{T} \hat{\Pi}_{\rho \sigma}^{T}}{p^{2}+\frac{n^{2}}{R^{2}}} \equiv \frac{\Pi_{\mu \nu \rho \sigma}\left(m_{n}\right)}{p^{2}+m_{n}^{2}}
$$

where $\hat{\Pi}_{\mu \nu}^{T}=\eta_{\mu \nu}-p_{\mu} p_{\nu} /\left(m_{n}\right)^{2}$.

Let us now focus on the zero modes. Notice that the radion mixes kinetically to the graviton. It is convenient to diagonalize the kinetic term via the Weyl shift $h_{\mu \nu}^{(0)} \equiv \bar{h}_{\mu \nu}-\frac{1}{2} \phi \eta_{\mu \nu}$, after which $\phi$ acquires a self kinetic term

$$
\frac{3}{2} M_{5}^{3} \pi R \phi \square \phi
$$

while $\bar{h}_{\mu \nu}$ has obviously the kinetic term of massless graviton. At this point we can gauge fix the residual $4 \mathrm{D}$ reparametrization and gauge symmetry by using respectively the de Donder and Feynman gauges

$$
2 M_{5}^{3} \pi R\left\{\left(\partial^{\mu} \bar{h}_{\mu \nu}-\frac{1}{2} \partial_{\nu} \bar{h}_{\mu}^{\mu}\right)^{2}-\left(\partial^{\mu} A_{\mu}\right)^{2}\right\} .
$$

On shell we have 2 physical helicity states in both $\bar{h}$ and $A$. These, including $\phi$, add up to 5 states: the same number we found at each excited level, but here they are shared among particles of different spin.

The presence of the radion $\phi$ makes this theory quite different from ordinary 4D Einstein gravity (the additional scalar is sometimes called a Brans-Dicke field). The tensor field that couples to ordinary 4D matter and thus describes the observable fluctuations of the $4 \mathrm{D}$ geometry is the original metric $h_{\mu \nu}^{(0)}$ and not $\bar{h}_{\mu \nu}$. Therefore the relevant graviton propagator is

$$
\begin{aligned}
\left\langle h_{\mu \nu}^{(0)} h_{\rho \sigma}^{(0)}\right\rangle & =\left\langle\bar{h}_{\mu \nu} \bar{h}_{\rho \sigma}\right\rangle+\frac{1}{4}\langle\phi \phi\rangle \\
& =\frac{1}{M_{4}^{2}}\left\{\frac{\frac{1}{2}\left(\eta_{\mu \rho} \eta_{\sigma \nu}+\eta_{\mu \sigma} \eta_{\rho \nu}\right)-\frac{1}{2} \eta_{\mu \nu} \eta_{\rho \sigma}}{q^{2}}\right. \\
& \left.+\frac{1}{6} \frac{\eta_{\mu \nu} \eta_{\rho \sigma}}{q^{2}}\right\}
\end{aligned}
$$


where the first term is just the result we would get in ordinary GR and the second contribution, proportional to $1 / 6$, is due to the radion. In the non-relativistic regime the effects of the tensor and scalar field are indistinguishable. The Newton constant is determined by $\left\langle h_{00}^{(0)} h_{00}^{(0)}\right\rangle$ and given by

$$
32 \pi G_{N}=\frac{2}{M_{4}^{2}}\left(\frac{1}{2}+\frac{1}{6}\right)=\frac{4}{3} \frac{1}{M_{4}^{2}}
$$

where we have indicated separately the contributions of the tensor and of the scalar. However in the relativistic regime the implications of the two terms are quite different. In particular $\phi$ does not couple to photons as they have a traceless energy momentum tensor. Now, one of the most accurate tests of GR is the measurement of the deflection of light by the gravitational field of the Sun: the experimental result agrees with the theory to about 1 part in $10^{3}$. In the theory at hand, $\phi$ does not contribute to this deflection, and the scattering angle, expressend in terms of the non-relativistic $G_{N}$ of eq. (1.47), is therefore only 3/4 of the GR prediction. This result is completely ruled out by the data. In order to meet consistency, $\phi$ should be given a mass $m_{\phi}$, so that its contribution to the potential will decay as $e^{-m_{\phi} r} / r$ and become quickly irrelevant for $r>1 / m_{\phi}$. Notice that giving a mass to $\phi$ corresponds to stabilizing the size of the 5 th dimension. The agreement between the value of $G_{N}$ measured in post Cavendish experiments [21-23] down to distances of order $100 \mu \mathrm{m}$ with that governing post Newtonian corrections in the solar system forces the mass of $\phi$ to be bigger that $\hbar c / 100 \mu m \sim 10^{-3}$ $\mathrm{eV}$.

To gain another viewpoint on the compactification of gravity from $D$ down to 4 dimensions, it is worth to count the physical states of gravity around ordinary D-dimensional (non-compact) Minkowsky space. The metric symmetric tensor $h_{M N}$ corresponds to $D(D-1) / 2$ fields. By using the $D$ gauge transformations $\epsilon_{N}$ we can eliminate $D$ of these fields. For instance we can go to the Gaussian normal gauge where all time components vanish $h_{00}=h_{0 i}=0$. This choice is the analogue of Coulomb gauge in Maxwell's theory. However as in any gauge theory, even after fixing the gauge, we must still impose the equations of motion of $h_{00}$ and $h_{0 i}$ as an initial time constraint

$$
\frac{\partial \mathcal{L}}{\delta h_{00}}=\nabla^{i} \nabla^{j} h_{i j}-\left.\nabla^{i} \nabla_{i} h_{j}^{j}\right|_{t=0}=0 \quad \frac{\partial \mathcal{L}}{\delta h_{0 i}}=\left.\left(\nabla^{j} \dot{h}_{i j}-\nabla_{i} \dot{h}_{j}^{j}\right)\right|_{t=0}=0
$$

where by the dot and by $\nabla_{i}$ we indicate respectively detivatives with respect to the time, $t$, and space, $x^{i}$, coordinates. The divergence of the 
$h_{i j}$ equations of motion

$$
\left(\nabla^{j} \ddot{h}_{i j}-\nabla_{i} \ddot{h}_{j}^{j}\right)=0
$$

ensures that both constraints remain valid at all times ${ }^{\S}$. To clarify things it is convenient to decompose $h_{i j}$ in spin components as previously done in eq. (1.42)

$$
h_{i j} \equiv H_{i j}^{T T}+\nabla_{i} V_{j}^{T}+\nabla_{j} V_{i}^{T}+\left(\delta_{i j}-\frac{\nabla_{i} \nabla_{j}}{\nabla^{2}}\right) H+\frac{\nabla_{i} \nabla_{j}}{\nabla^{2}} V
$$

Moreover it should be noticed that there is a residual gauge freedom preserving the Gaussian normal condition

$$
\epsilon_{0} \equiv \epsilon_{0}\left(x_{i}\right) \quad \epsilon_{i} \equiv \epsilon_{i}^{T}\left(x_{i}\right)-\nabla_{i}\left(\epsilon_{L}\left(x_{i}\right)+t \epsilon_{0}\left(x_{i}\right)\right) .
$$

Notice that the $\epsilon$ 's depend only on the space coordinates and that $\nabla^{i} \epsilon_{i}^{T}=$ 0 . Now, the divergence and trace of the $h_{i j}$ equation of motion imply

$$
\nabla_{i} \ddot{H}=\nabla^{2} \ddot{V}_{i}^{T}=0 \quad \ddot{V}+(D-2) \ddot{H}-(D-3) \nabla^{2} H=0 .
$$

Assuming that our fields $F$ vanish fast enough at spacial infinity, $\nabla^{2} F=$ 0 implies $F=0$. The first two equations then imply $H=H_{0}\left(x_{i}\right)+$ $t H_{1}\left(x_{i}\right)$ and $V_{j}^{T}=V_{0 j}^{T}\left(x_{i}\right)+t V_{1}^{T}\left(x_{i}\right)$. The initial time constraints imply however $H_{0}=H_{1}=V_{1 j}^{T}=0$. In turn eq. (1.52) implies $V=V_{0}\left(x_{i}\right)+$ $t V_{1}(x)$. At this point we are left with $D$ functions $V_{0 i}^{T}, V_{0}, V_{1}$ which can be completely eliminated by the residual gauge freedom $\epsilon_{i}^{T}, \epsilon_{L}, \epsilon_{0}$. Notice that the initial time constraints eliminate 1 dynamical variable, $H$, plus $D-1$ "velocities" $\dot{H}, \dot{V}_{i}^{T}$. Instead the gauge freedom allows to eliminate $D-1$ variables and 1 velocity. This generalizes the situation in electromagnetism where the Gauss constraint eliminates 1 velocity, $\nabla^{i} \dot{A}_{i}$, while the residual gauge freedom eliminates $\nabla^{i} A_{i}$.

The result of all this is that, after going to the $h_{00}=h_{0 i}=0$ gauge, an additional $D$ deegrees of freedom are eliminated $\left(V_{i}^{T}, H, V\right)$ and we are left with $D(D-1) / 2-2 D=D(D-3) / 2$ propagating fields, corresponding to $H_{i j}^{T T}$.

Before concluding this section we discuss the more general case of Einstein gravity in $D=4+n$ dimensions, with the $n$ extra dimensions compactified on a square torus $T^{n}[15]$. Indicating by $i$ and $x^{i}$ the extra

\footnotetext{
$\S$ Again this is analogous to electromagnetism. In Coulomb gauge, the $A_{0}$ equation of motion constraint gives Gauss law $\vec{\nabla} \cdot \vec{E}=0$ at initial times. Maxwell's equation $\dot{\vec{E}}=\vec{\nabla} \times \vec{B}$ implies the validity of Gauss's law at all times.
} 


\begin{tabular}{|c|c|c|c|c|}
\hline field & $\begin{array}{c}\text { original } \\
\text { d.o.f }\end{array}$ & $\begin{array}{c}(-) \text { gauge } \\
\text { fixing }\end{array}$ & $(-)$ eqs. motion & $\begin{array}{c}\text { propagating } \\
\text { d.o.f. }\end{array}$ \\
\hline$(J=2) h_{\mu \nu}$ & 10 & 0 & -5 & 5 \\
\hline$(J=1) h_{\mu i}$ & $4 n$ & -4 & $-(n-1)$ & $3(n-1)$ \\
\hline$(J=0) h_{i j}$ & $\frac{n(n+1)}{2}$ & $-n$ & 0 & $\frac{n(n-1)}{2}$ \\
\hline
\end{tabular}

Table 1. Number of degrees of freedom (d.o.f.) off-shell and on-shell for each field component

indices and coordinates, $T^{n}$ is defined by the equivalence relation $x^{i} \sim$ $x^{i}+2 \pi R n^{i}$ with $n^{i}$ a vector with integer entries. KK levels are labelled by a vector of integers $(\vec{n})_{i}=n_{i}$ associated to the momentum $n_{i} / R$ along the $T^{n}$ directions. The counting of physical degrees of freedom for each massive KK level is shown in Table (1). It is easy to check that gauge invariance allows to eliminate the $4 \mathrm{D}$ vector $n^{i} h_{i \mu}$ (4 fields) and the scalars $n^{i} h_{i j}$ (n fields). On shell $n-1$ longitudinal components from the remaining $n-1$ vectors and 5 more components from $h_{\mu \nu}$ are eliminated. The propagating degrees of freedom are correspondingly $n-1$ massive vectors and 1 massive graviton. Thus there finally result $(n+4)(n+1) / 2=D(D-3) / 2$ physical states, in agreement with our previous derivation. At the zero mode level there is the same number of degrees of freedom, but they are shared among 1 graviton $h_{\mu \nu}^{(0)}, 2 n$ graviphotons $A_{\mu}^{i}$ and a symmetric matrix of $n(n+1) / 2$ scalars $\phi_{i j}$. The scalars $\phi_{i j}$ are the moduli describing the fluctuations in the shape and size of the torus. In particular the trace $\phi_{i}^{i}$ describes the fluctuations of the torus volume. This field mixes to the $4 \mathrm{D}$ graviton leading to a propagator with an extra scalar term

$$
\frac{1}{M_{4}^{2}}\left\{\frac{\frac{1}{2}\left(\eta_{\mu \rho} \eta_{\sigma \nu}+\eta_{\mu \sigma} \eta_{\rho \nu}\right)-\frac{1}{2} \eta_{\mu \nu} \eta_{\rho \sigma}}{q^{2}}+\frac{n}{2 n+4} \frac{\eta_{\mu \nu} \eta_{\rho \sigma}}{q^{2}}\right\}
$$

In order to agree with observations, the volume modulus should be stabilized.

\subsection{Large Extra-Dimensions}

A very interesting arena where to apply the concepts that we introduced is given by the scenario of large extra dimensions. This scenario has been advocated by Arkani-Hamed, Dimopoulos and Dvali (ADD) as an alternative viewpoint on the gauge hierarchy problem [2]. With respect to the standard picture for physics beyond the SM the ADD proposal represents a dramatic shift of paradigm. In the standard scenario, fundamental interactions are described by an ordinary quantum 
field theory up to energy scales larger that the Grand Unification scale $10^{16} \mathrm{GeV}$. Above this scale quantum gravity effects or string theory imply a radical revision of fundamental physics. According to the ADD proposal, instead, this radical revision is needed right above the weak scale! The proposal is specified by three main features

- There exists a number of $n$ new spacial compact dimensions. For instance a simple manifold could be just $\mathcal{M}_{4} \times T^{n}$.

- The fundamental Planck scale of the theory is very low $M_{D} \sim$ $\mathrm{TeV}$.

- The SM degrees of freedom are localized on a 3D-brane stretching along the 3 non-compact space dimensions.

As we will now explain, these three requirement allow for a drastically different viewpoint on the hierarchy problem, without leading to any stark disagreement with experimental observations. Let us focus on gravity first. As we have already seen in the simple case of KaluzaKlein's theory, the macroscopic Planck mass $M_{4}^{2}$ of the effective $4 \mathrm{D}$ theory is related to the microscopic $M_{D}$ via

$$
M_{4}^{2}=M_{D}^{2+n} V_{n}
$$

where $V_{n}$ is the compactification volume. For a torus we have $V_{n}=$ $(2 \pi R)^{n}$ and the above result follows from a simple generalization of the analysis we previously did on $S_{1}$. We can also obtain this relation by considering directly the effective action for a purely zero mode $g_{\mu \nu}\left(x^{\mu}, x^{i}\right) \equiv \bar{g}_{\mu \nu}\left(x^{\mu}\right)$ fluctuation of the metric along $\mathcal{M}_{4}$

$$
2 M_{D}^{2+n} \int d^{4} x^{\mu} d^{n} x^{i} \sqrt{g} \mathcal{R}_{D}(g) \quad \leftrightarrow \quad 2 M_{D} V_{n} \int d^{4} x^{\mu} \sqrt{\bar{g}} \mathcal{R}_{4}(\bar{g})
$$

where we have explicitly indicated the dimensionality of the Ricci tensor. The main remark of ADD is based on eq. (1.54). Provided the volume of compactification is large enough, even a low gravity scale $M_{D}$ can reproduce the physical value $M_{4}=2 \times 10^{18} \mathrm{GeV}$. Before discussing the needed size of $R$, notice that eq. (1.54) has a very simple interpretation via Gauss's theorem. Consider the Newtonian potential $\varphi \equiv h_{00} / 2$ generated by a test mass $M$ in the linearized approximation. At a distance $r \ll R$ the compactness of the extra dimensions does not play a relevant role: the potential is to a good approximation $S O(3+n)$ symmetric and given by

$$
\left.\varphi\right|_{r \ll R} \simeq-\frac{\Gamma\left(\frac{n+3}{2}\right)}{(2 n+4) \pi^{\frac{3+n}{2}}} \frac{1}{M_{D}^{2+n}} \frac{M}{r^{1+n}} .
$$




\begin{tabular}{|c|c|}
\hline$n$ & $R$ \\
\hline \hline 1 & $6 \times 10^{13} \mathrm{~cm}$ \\
\hline 2 & $0.4 \mathrm{~mm}=1 /\left(10^{-4} \mathrm{eV}\right)$ \\
\hline 4 & $10^{-8} \mathrm{~mm}=1 /(20 \mathrm{KeV})$ \\
\hline 6 & $2.5 \times 10^{-11} \mathrm{~mm}=1 /(10 \mathrm{MeV})$ \\
\hline
\end{tabular}

Table 2. Radius of compactification for fixed value of $M_{D}^{G R W}=1 \mathrm{TeV}$, where $\left(M_{D}^{G R W}\right)^{2+n} \equiv 4(2 \pi)^{n} M_{D}^{2+n}$ is the Planck mass defined in the first paper of ref. [15].

as dictated by Gauss's theorem. At $r \gg R$ the field lines stretch along the 3 non-compact directions, the potential is only $S O(3)$ symmetric. The surface encompassing the field flux is now the two sphere (noncompact directions) times the compactification manifold; for instance $S_{2} \times T^{n}$. Applying Gauss's theorem we find then

$$
\left.\varphi\right|_{r \gg R} \simeq-\frac{n+1}{16 \pi(n+2) M_{D}^{2+n} V_{n}} \frac{M}{r} \equiv \frac{n+1}{16 \pi(n+2)} \frac{1}{M_{4}^{2}} \frac{M}{r}
$$

from which we recover again eq. (1.54). In practice the large distance field is made weaker by the large extradimensional volume in which the field lines can spread. (The dependence of eq. (1.57) on $n$ is due to the massless radion. For $n=1$ it agrees with eq. (1.47), while for general $n$ eq. (1.57) is simply the Fourier transform of eq. (1.53).).

If the ultimate cut-off $M_{D}$ is of order the weak scale itself $G_{F}^{-1 / 2}$, then the expected quantum corrections to the Higgs mass are of the order of its phenomenologically favored value $m_{H} \sim G_{F}^{-1 / 2}$. In this respect the hierarchy problem, in its ordinary formulation, is practically eliminated when $M_{D} \sim 1 \mathrm{TeV}$. With this input, and with the observed value of $M_{4}$, eq. (1.54) predicts the size of $V_{n}$. In Table 2, we give the radius of compactification in the case of a square n-torus. We stress, see eqs. $(1.56,1.57)$, that Newton's law is reproduced only at distances larges than $R$. The case $n=1$ requires a radius of compactification of the size of the solar system, which is largely ruled out. However already for $n$ greater or equal than 2 the resulting radius is not unreasonable. Indeed experimental tests of gravity at distances shorter than a millimeter are extremely arduous. This is largely due to the presence of Van der Waals forces, which tend to swamp any interesting measurement. At present the best bound relegates $O(1)$ deviations from Newton's law (the ones we would expect is our scenario at $r \lesssim R$ ) to distances shorter than $200 \mu \mathrm{m}$. In this respect the case $n=2$ is not barely inconsistent. $n=2$ is also experimentally interesting, as it predicts deviations in the range of 
present sensitivities. The search for deviations from Newton's law is an active experimental field, also greatly stimulated by the ADD proposal.

Focusing on gravity only, we have shown that for $n \geq 2$ the radius of compactification is small enough. On the other hand the Standard Model as been verified down to distances much shorter than the radii shown in the table. The SM is a $3+1$ dimensional quantum field theory and its predictions depend crucially on this property. LEP, SLC and Tevatron have tested the SM up to an energy of order $1 \mathrm{TeV}$, corresponding to a distance of order $10^{-16} \mathrm{~cm}$. Experimentally then, the $\mathrm{SM}$ is a $3+1$ dimensional system down to a distance much shorter that the radius of compactification. Localizing all the SM degrees of freedom on a 3-brane is an elegant way to realize this experimental fact, while keeping larger radii of compactification. Now it will be the brane size, or whatever other characteristic brane cut-off scale, perhaps $1 / M_{D}$ itself, to characterize the length scale down to which the SM is a valid effective field theory. This scale can conceivably be $\gtrsim 1 \mathrm{TeV}$. For instance, the ADD scenario could be realized in type I string theory $[16,17]$ with the SM localized on a D-brane. In this case the string scale $M_{S}$, governing the mass of Regge resonances, acts as UV cut off of the brane effective theory.

This completes the basic description of the ADD scenario. It must however be said that, as it stands, the ADD proposal is a reformulation of the hierarchy problem and not yet a solution [18]. Instead of the small Higgs vacuum expectation value (VEV) of the old formulation, we now need to explain why the compactification volume $V_{n}$ is so much bigger that its most natural scale $1 / M_{D}^{n}$ :

$$
V_{n} M_{D}^{n} \sim 10^{33}
$$

$V_{n}$, or equivalently the radius $R$, is a dynamical degree of freedom, a scalar field. We have already shown that in the case of $T^{n}$ the fluctuation of $V_{n} \equiv(2 \pi R)^{n}$ corresponds at linear order to the trace $h_{i}^{i}$. Since we want a large $\langle R\rangle$ the scalar potential $V(R)$ will have to be much flatter than naively expected at large values of $R$. As far as we know, the most natural way to achieve such flat potentials is by invoking supersymmetry. So, if the ADD scenario is realized in Nature it is likely to be so together with supersymmetry at some stage. Notice that in the conventional formulation of the hierarchy problem supersymmetry is invoked to ensure a flat potential at small values of the Higgs field, i.e. a small Higgs mass. As a matter of fact, the ADD proposal maps a small VEV problem into a basically equivalent large VEV problem. In the new scenario the hierarchy problem has become a sort of cosmological constant problem. Indeed a vacuum energy density $\Lambda^{4+n}$ would add to the radius potential a term $\sim \Lambda^{4+n} R^{n}$. This grows very fast at large $R$ so we expect [18] that 
$\Lambda^{4+n}$ should be much smaller than its natural value $(\mathrm{TeV})^{4+n}$. In this respect the presence of bulk supersymmetry would be a natural way to enforce a small $\Lambda^{4+n}$, thus helping to explain the large volume. Indeed ref. [19] presents a simple mechanism which produces large radii at $n=2$, but which works for a vanishing bulk cosmological constant $\Lambda^{6}$. Although the model considered is not supersymmetric it is conceivable that the same mechanism will generalize to a supersymmetry set-up and thus lead to a truly natural generation of the hierarchy.

One reason why the ADD proposal is important is theoretical. The hope is that such a drastic revision of our view of fundamental interactions may open the way to new solutions to old problems, like the cosmological constant problem for instance. Having string theory right at the weak scale may also end up being the right ingredient to build the right string model. However none of these breakthroughs has come yet. The interest in the ADD proposal is at the moment associated to its potentially dramatic phenomenological implications [20]. There are two classes of laboratory tests of large extra-dimensions. We have already commented on the first class, the search for deviations from Newton's law at short but macroscopic distances. This is done in table top experiments. These deviations could be determined by the light moduli, like the radius $R$ [18], or by the lowest Kaluza-Klein (KK) $\mathrm{J}=2$ modes. Another source of deviation could be the lowest KK mode of a bulk vector field gauging baryon number [20]. At present, $O(1)$ deviations from Newton's law have been excluded down to a length $\sim 200 \mu \mathrm{m}$ [21], while forces that have a strength $>10^{4}$ of gravity are bounded to have a range smaller than $20 \mu \mathrm{m}[22,23]$. Notice that this class of effects crucially depends on the features of the compactification manifold at large "lengths", as they determine the masses of the lightest modes. For instance the presence of even a small curvature of the compactification manifold can drastically affect these prediction by lifting the lightest states. On dimensional grounds, if the typical curvature length is $L$ the modes with mass $<1 / L$ will be affected and possibly made heavier.

The second class of tests is given by high energy collisions [15]. In this case we deal with either gravitons at virtuality $Q \gg 1 / R$ or with real gravitons measured with too poor an energy resolution to distinguish individual KK levels. In practice, for high energy processes happening on a short time scale $\tau \sim 1 / E \ll R$, causality and locality imply that we cannot notice that the extra dimensions are compact. Therefore we can take the limit $R \rightarrow \infty$ and work as if our brane were embedded in infinite $(4+n)$-dimensional Minkowski space. (If the compactification manifold had curvature length $L \ll R$, then the same reasoning would apply for energies $E \gg 1 / L)$. Moreover at energy $E<M_{D}$, as discussed in section 


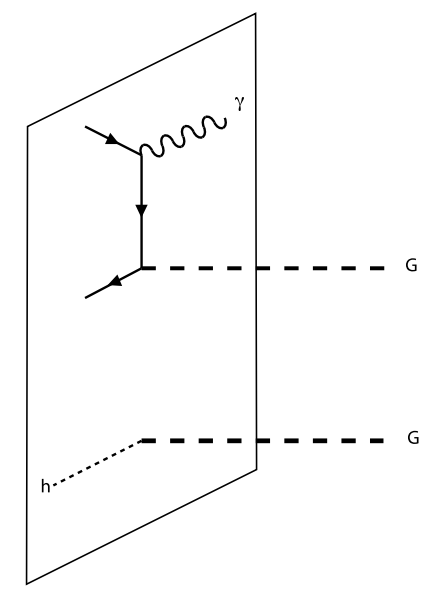

Figure 7. Example of two processes with missing energy by bulk graviton radiation.

1.1.4, we can reliably compute the amplitudes in a systematic derivative expansion. The characteristic signals are then associated to the emission of gravitons $(G)$ which escape undetected into the extra dimensions. Interesting examples (see the figure) are given by the processes $e^{+} e^{-} \rightarrow$ $\gamma+G=\gamma+\not E$ or $p p \rightarrow$ jet $+\mathbb{E}$ or possibly by the invisible decay of the Higgs into just one graviton [24]. The latter process does not violate the conservation of angular momentum, since there are KK gravitons of spin 0 . The relevant interactions are obtained by expanding the brane action for the SM (as in eq. (1.9)) in powers of the induced metric. For processes with the emission of one graviton we have just the energy momentum term

$$
\mathcal{L}_{\text {int }}=-\frac{1}{2} T_{\mu \nu}^{S M} h^{\mu \nu}\left(x, y^{i}=0\right)
$$

where we have assumed the brane to be located at $y^{i}=0$. Emission rates can be computed by expanding $h_{\mu \nu}\left(x, y_{i}=0\right)$ in KK modes. For instance, the differential cross section for $e^{+} e^{-} \rightarrow \gamma G$

$$
\begin{gathered}
\frac{d^{2} \sigma}{d x_{\gamma} d \cos \theta}\left(e^{+} e^{-} \rightarrow \gamma G\right)=\frac{\alpha}{128} \frac{1}{(4 \pi)^{\frac{n}{2}} \Gamma\left(\frac{n}{2}\right)}\left(\frac{s^{\frac{n}{2}}}{M_{D}^{n+2}}\right) \\
{\left[F_{n}\left(x_{\gamma}, \cos \theta\right)+O\left(\frac{s}{M_{D}^{2}}\right)\right]} \\
F_{n}(x, y)=\frac{2(1-x)^{\frac{n}{2}-1}}{x\left(1-y^{2}\right)}\left[(2-x)^{2}\left(1-x+x^{2}\right)-3 y^{2} x^{2}(1-x)-y^{4} x^{4}\right] .
\end{gathered}
$$


Here $x_{\gamma}=2 E_{\gamma} / \sqrt{s}, E_{\gamma}$ is the photon energy, and $\theta$ is the angle between the photon and beam directions. At leading order in $E / M_{D}$ this process is predicted just in terms of one new parameter, $M_{D}$ itself. Higher order corrections will depend on new operator coefficients. Similar results can be found for all the other processes [15].

Notice that graviton emission violates the conservation of momentum along the $y$ directions. This is not surprising since the presence of the brane at $y=0$ breaks translation invariance. However one might worry that non-conservation of the brane energy momentum might lead to inconsistencies in the interaction with gravity; after all Einstein equations demand energy momentum conservation. But this is not the case. Even though the global momentum $P_{i}$ along $y_{i}$ is not conserved (or better not defined) the full energy momentum tensor $T_{M N}$ for the brane plus matter is indeed locally conserved. Conservation of a local current in the absence of a globally conserved charge is the landmark of spontaneous symmetry breaking, and is precisely what happens here. As we have said before, translations in $y^{i}$ are a non-linearly realized symmetry, with the branons $Y^{i}$ acting as Goldstone bosons. Local conservation of momentum implies the presence of the branons and hence their production in fundamental processes. Since the brane is infinitely massive it cannot undergo a global recoil, but conservation of its energy momentum tensor implies the possibility of local recoil by branon emission. The emission of branons is another possible signature of the braneworld scenario. Branons $Y$ are emitted in pairs. At lowest order one finds [25]

$$
\frac{d^{2} \sigma}{d x_{\gamma} d \cos \theta}\left(e^{+} e^{-} \rightarrow \gamma Y Y\right)=\frac{\alpha}{1920 \pi^{5}}\left(\frac{s^{3}}{f^{8}}\right)\left[F_{6}\left(x_{\gamma}, \cos \theta\right)+O\left(\frac{s}{M_{D}^{2}}\right)\right]
$$

which, up to an overal constant, is the same result one obtains for graviton emission at $D=10$. A similar result is obtained in the case of hadronic collisions. Comparing eqs. $(1.60,1.62)$ to experiments one obtains experimental bounds of the scales $M_{D}$ and $f$. As the effect grows with $\sqrt{s}$ the best bounds are obtained from the higher energy experiments, LEP2 and Tevatron. In particular LEP2 implies the bound $f>100 \mathrm{GeV}$ [25]. The combined LEP Tevatron bounds on $M_{D}$ [27]are shown in Table 3 . These direct bounds are not very strong. The reason is that the cross section is suppressed by a rather large power of $E$ over the high scale. The LHC will be a better machine to test these ideas through the direct production of gravitons or string states [15, 26]. At present stronger bounds come from other effects, associated to contact 4 fermion interactions, that are expected in the ADD scenario model without being a distinctive feature. The translation of the bounds from 


\begin{tabular}{|c|c|c|c|c|c|}
\hline$n$ & 2 & 3 & 4 & 5 & 6 \\
\hline \hline$M_{D}^{G R W}(\mathrm{TeV})$ & $>1.45$ & $>1.09$ & $>0.87$ & 0.72 & 0.65 \\
\hline
\end{tabular}

Table 3. Collider bounds on $M_{D}^{G R W}$

these other effects into bounds on $M_{D}$ is a model dependent procedure, but it is fair to say that they roughly imply $M_{D} \gtrsim 3 \mathrm{TeV}$ [27]. Finally we should mention that, with enough luck, the LHC may also study gravitational scattering at energies in excess of the Planck mass $M_{D}$, the so-called transplanckian regime. For $M_{D} \sim 1-3 \mathrm{TeV}$, the most energetic collisions at LHC, at $\sqrt{s}=14 \mathrm{TeV}$, should start manifesting the transplanckian dynamics, which consists of black-hole [28] or string ball [29] production and also of the characteristic gravitational elastic scattering [30]. The common features of these processes is to give cross section at high energy, and fixed angle, that asymptotically grow like a power of energy. This would be an undisputable signal that the high energy dynamics of gravity, a force whose associated charge is energy itself, has been detected.

Computations like those we have outlined are relevant also to study the cosmological and astrophysical implications of the ADD scenario. The phenomenology of these models is now a very wide field. Unfortunately in order to cover it appropriately we would be lead outside the main goal of the present lectures, which concerns the basic physics principles and tools. Excellent pedagogical reviews of recent results with extensive references are given by refs. [31, 32].

\section{Part II: the Randall-Sundrum model}

In the second part of these lecture we will focus on a specific model proposed by Randall and Sundrum (RS) which is interesting both from the theoretical and the pedagogical viewpoint. On one side the RS model realizes a new way of approaching the hierarchy problem, on the other it is simple enough to allow a number of rather instructive computations. Moreover it naturally demands the introduction of the concept of holography, the basis of the $A d S / C F T$ correspondance [33, 34]. All these reasons make the RS model a very rich and instructive lecture subject.

\section{$2.1 \quad$ The Model}

Let us consider a model with a 5 th dimension and let us compactify it by considering the following equivalence relations for the fifth coordinate 


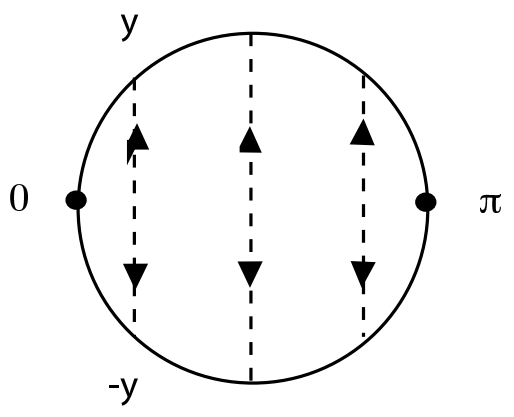

Figure 8. Graphical representation of $S_{1} / Z_{2}$.

$y$

$$
y \sim y+2 \pi \quad y \sim-y .
$$

The first relation, alone, would define a circle $S_{1}$. The second relation, a $Z_{2}$ reflection, implies identification of opposite points on the circle, as shown in the Fig. (8). $y=0$ and $y=\pi$ are fixed points under $Z_{2}$ on the circle and are identified with themselves. The resulting space from this identifications is called a $S_{1} / Z_{2}$ orbifold. $S_{1} / Z_{2}$ is equivalent to the $[0, \pi]$ segment, but for computational purposes it is useful work with the full $S_{1}$ covering space with $Z_{2}$ identification. Consider now the metric on this space

$$
d s^{2}=g_{\mu \nu} d x^{\mu} d x^{\nu}+2 g_{\mu 5} d x^{\mu} d y+g_{55} d y^{2} .
$$

The points related by $y \rightarrow-y$ are physically identical, and under $Z_{2}$ the interval $d s^{2}$ should be invariant. Since $d y \rightarrow-d y$ under $Z_{2}$, we then have

$g_{\mu \nu}(x, y)=g_{\mu \nu}(x,-y) \quad g_{55}(x, y)=g_{55}(x,-y) \quad g_{\mu 5}(x, y)=-g_{\mu 5}(x,-y)$.

From the last identity and by the continuity of $g_{\mu 5}$ it follows that $g_{\mu 5}(x, 0)$ $=g_{\mu 5}(x, \pi)=0$. Changes of coordinates

$$
\bar{x}^{\mu}=\bar{x}^{\mu}(x, y) \quad \bar{y}=\bar{y}(x, y)
$$

should still parametrize an orbifold and respect eq. (2.1). Without loss of generality we can impose $\bar{y}$ to be the new orbifold coordinate satisfying

$$
\bar{y}(x, y+2 \pi)=\bar{y}(x, y)+2 \pi \quad \bar{y}(x,-y)=-\bar{y}(x, y)
$$

generalizing what we did before for the circle. Notice that under reparametrizations the fixed point are mapped onto themselves $\bar{y}(x, 0)=0$, 
$\bar{y}(x, \pi)=\pi$ At the boundaries, eq. (2.4) reduces to $4 \mathrm{D}$ reparametrizations of the boundaries themselves

$$
\begin{array}{ll}
0) & x^{\mu} \rightarrow \bar{x}^{\mu}(x, 0) \\
\pi) & x^{\mu} \rightarrow \bar{x}^{\mu}(x, \pi)
\end{array}
$$

under which the induced metrics

$$
g_{0 \mu \nu} \equiv g_{\mu \nu}(x, 0) \quad g_{\pi \mu \nu} \equiv g_{\mu \nu}(x, \pi)
$$

are covariant tensors. Now, using $g_{0, \pi}$ we can write the most general invariant action involving also fields and interactions localized at the boundaries

$$
\begin{aligned}
S & =\int d^{4} x \int_{0}^{2 \pi} d y\left\{\sqrt{g}\left[2 M_{5} \mathcal{R}(g)-\tau\right]\right. \\
& \left.+\delta(y) \sqrt{g}_{0}\left[\mathcal{L}_{0}-\tau_{0}\right]+\delta(y-\pi) \sqrt{g}_{\pi}\left[\mathcal{L}_{\pi}-\tau_{\pi}\right]\right\}
\end{aligned}
$$

where $\tau$ and $\tau_{0}, \tau_{\pi}$ are respectively the bulk cosmological constant and boundary tensions. By $\mathcal{L}_{0, \pi}$ we indicate any other interactions involving fields localized at the boundary. Neglecting the latter, the 5D Einstein equations are

$$
\begin{gathered}
\sqrt{g} G_{M N}= \\
\frac{-1}{4 M_{5}^{3}}\left[\tau \sqrt{g} g_{M N}+\left(\tau_{0} \sqrt{g}_{0} g_{0 \mu \nu} \delta(y)+\tau_{\pi} \sqrt{g}_{\pi} g_{0} \pi_{\mu \nu} \delta(y-\pi)\right) \delta_{M}^{\mu} \delta_{N}^{\nu}\right]
\end{gathered}
$$

We look for a solution with the following Poincaré invariant ansatz

$$
d s^{2}=e^{-2 \sigma(y)} \eta_{\mu \nu} d x^{\mu} d x^{\nu}+r_{c}^{2} d y^{2}
$$

where $r_{c}$ is a parameter with dimension [length] parametrizing the proper distance between the two fixed points. The $\mu 5$ equation is identically satisfied while the 55 is

$$
\left(\frac{\sigma^{\prime}}{r_{c}}\right)^{2}=-\frac{\tau}{24 M_{5}^{3}} \equiv k^{2}
$$

ฯ On the segment $[0, \pi]$ these are the reparametrizations that do not move the boundaries. One could allow more general reparametrizations under which the boundary points are shifted. The resulting field space would be obviously larger. The physics would however not be affected. From the point of view of this more general formulation our field space is obtained just by a partial gauge fixing. 
implying a negative cosmological constant $\tau<0$. Imposing the orbifold symmetry eq. (2.11) has two solutions (up to trivial coordinate changes):

$$
\sigma(y)= \pm k r_{c}|y|
$$

Finally the $\mu \nu$ equation is

$$
\sigma^{\prime \prime}=\frac{r_{c}}{12 M_{5}^{3}}\left[\tau_{0} \delta(y)+\tau_{\pi} \delta(y-\pi)\right]
$$

which is solved by eq. (2.12) provided the following conditions hold

$$
\tau_{0}=-\tau_{\pi}= \pm 24 M_{5}^{3} k
$$

Without loss of generality, we can choose the solution $\sigma=+k r_{c}|y|$. Notice that the tension $\tau_{\pi}$ is negative. We will later show that this does not lead to instabilities. Eq. (2.14) represents a tuning of two parameters, in the absence of which there would not exist a static solution with Poincaré symmetry. This does not seem at all a desired feature for a model aiming at a solution of the gauge hierarchy problem! The meaning of eq. (2.14) will become more clear below. We will then explain that in the complete model there remains just one tuning, the one associated to the $4 \mathrm{D}$ cosmological constant. This is a situation common to all the other solutions of the gauge hierarchy problem, like supersymmetry or technicolor.

Our orbifold with metric

$$
d s^{2}=e^{-2 k r_{c}|y|} d x_{\mu} d x^{\mu}+r_{c}^{2} d y^{2}
$$

corresponds to two slices of 5-dimensional anti-de Sitter space (AdS5) glued back to back at the fixed points. The full AdS space is obtained by $|y| \rightarrow y$ and for $y \in(\infty,+\infty) . k^{2}=\mathcal{R}_{5}(g)$ parametrizes the curvature of this space. In order for our effective field theory to be a valid description it should be $k \ll M_{5}$. A region with fixed $x$ coordinates exported along $y$ describes a throat that gets exponentially narrow at larger $y$. Fig. 9 depicts the same contraction for the wavelength of infalling quanta, which we will later discuss in more detail. It is sometimes said that the space is "warped" by the $y$ dependent factor multiplying the $4 \mathrm{D}$ metric.

Our metric can be used to Redshift 4-dimensional mass parameters as suggested at the beginning of these lectures. Assume we place at 0 and $\pi$ two identical copies of a 4D QFT. Exactly like with two atoms in the gravitational field of a star, any direct experimental comparison of the masses of the equivalent states at each brane gives

$$
\frac{m_{\pi}}{m_{0}}=e^{-k r_{c} \pi}
$$




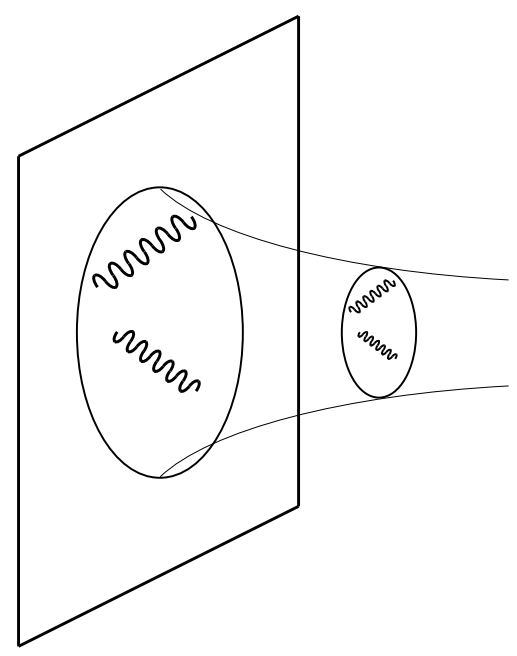

Figure 9. Contraction of wavelengths as particles fall through the AdS throat.

For $e^{-k r_{c} \pi} \sim m_{Z} / M_{4}$ this effect could be relevant in explaining the weak scale hierarchy. Because of the relative shift of mass scales the 0 and $\pi$ fixed points are called respectively the Planck and $\mathrm{TeV}$ brane. Notice also that, because of the appearance of an exponential factor, a Redshift of order $m_{z} / M_{4} \sim 10^{-16}$ is already obtained when the proper radius of compactification $r_{c} \pi$ is only about 35-40 times larger than the curvature radius $1 / k$. The latter could in turn be not much bigger than the $5 \mathrm{D}$ Planck length $1 / M_{5}$. Therefore a large hierarchy can be obtained from a rather small fifth dimension.

\subsection{Low energy effective theory}

The RS model is a generalization of the Kaluza-Klein theory that we already studied. It is then straightforward for us to discuss its zero mode content and to derive the low energy effective field theory. The main difference with respect to gravity on $S_{1}$, is that $g_{\mu 5}$ is odd under orbifold parity, see eq. (2.3). Then $g_{\mu 5}$ does not have a graviphoton zero mode and we can go to a gauge where $g_{\mu 5} \equiv 0$. On the other hand the graviton and radion zero modes are just obtained by promoting the Lorentz metric $\eta_{\mu \nu}$ and radius $r_{c}$ to 4-dimensional fields [3]

$$
d s^{2}=G_{M N} d X^{N} d X^{M}=e^{-2 k T(x)|y|} \bar{g}_{\mu \nu}(x) d x^{\mu} d x^{\nu}+T(x)^{2} d y^{2} .
$$

Notice indeed that when $\bar{g}_{\mu \nu}$ and $T$ are constant over space-time, eq. (2.17) is a solution of the equations of motion. $\left(T=r_{c}\right.$ is not fixed by 
the solution, while the solutions with arbitrary constant $\bar{g}_{\mu \nu}$ is simply obtained from the original solution by applying the coordinate change, $x^{\mu} \rightarrow A_{\nu}^{\mu} x^{\nu}$, with $A_{\mu}^{\rho} A_{\rho \nu}=\bar{g}_{\mu \nu}$.) Then the $5 \mathrm{D}$ action is stationary over the field manifold of constant $\bar{g}_{\mu \nu}$ and $T$, i.e. there is no "potential" for $\bar{g}_{\mu \nu}$ and $T$, so that they must correspond to massless particles. By substituting the ansatz in eq. (2.17) in the action we find indeed

$$
\mathcal{L}_{4}^{e f f}=\int \mathcal{L}_{5}(\bar{g}, T) d y=\left(\frac{M_{5}}{k}\right)^{3} \sqrt{\bar{g}}\left\{\left(2 k^{2}-2 \mu^{2}\right) \mathcal{R}(\bar{g})+12(\partial \mu)^{2}\right\}
$$

where

$$
\mu(x)=k e^{-k T(x) \pi} .
$$

and where all the metric contractions are done with $\bar{g}_{\mu \nu}$. This Lagrangian correctly describes the interactions of the zero modes up to terms with more than two derivatives. We will explain this in more detail when deriving the KK spectrum. By substituting $\langle T\rangle=r_{c}$ in the above equations we can read the effective 4-dimensional Planck scale

$$
M_{4}^{2}=\frac{M_{5}^{3}}{k}\left(1-e^{-2 k r_{c} \pi}\right) .
$$

By working with $T \equiv r_{c}$, eq. (2.17) substituted in the 5D Einstein term gives $\sqrt{g} \mathcal{R}_{5}(g)=\sqrt{\bar{g}} r_{c} e^{-2 \sigma(y)} \mathcal{R}_{4}(\bar{g})+\ldots$ Eq. (2.20) then simply corresponds to the integral

$$
M_{4}^{2}=M_{5}^{3} \int_{-\pi}^{\pi} e^{-2 \sigma(y)} r_{c} d y .
$$

The crucial aspect of this result is that $M_{4}$ is completely dominated by the region close to the Planck brane, where the warp factor is of order 1. Therefore the value of $M_{4}$ is insensitive to the Redshift of mass scales that takes place in the bulk and which is maximal at the $\mathrm{TeV}$ brane.

Now that we have calculated the Planck mass we can discuss the issue of the gauge hierarchy in more detail. Assume we localize all the fields of the Standard Model on the $\mathrm{TeV}$ brane. We indicate collectively by $H, \psi_{\alpha}$, and $A_{\mu}$ the scalars, fermions and vectors, and by $m$ the mass parameters (any mass parameter, including the Higgs mass). According to the ansatz of eq. (2.17), and keeping the radion fixed for simplicity, the induced metric at the $\mathrm{TeV}$ brane is

$$
g_{\pi \mu \nu}=e^{-2 \sigma(\pi)} \bar{g}_{\mu \nu}(x) .
$$

so that the low energy effective action including the $y=\pi$ boundary contribution is

$$
\mathcal{L}^{e f f}=\left\{\frac{2 M_{5}^{3}}{k}\left(1-e^{-2 \sigma(\pi)}\right) \sqrt{\bar{g}} \mathcal{R}(\bar{g})+\sqrt{g_{\pi}} L_{\pi}\left(g_{\pi \mu \nu}, H, \psi_{\alpha}, \mathcal{A}_{\mu}, m\right)\right\} .
$$


Notice that the metric that couples to matter is rescaled with respect to the one that appears in the Einstein term, as if different length units were used in the two actions. To make physics manifest it is useful to perform a constant Weyl transformation on the matter action. This is just a reparametrization of field variables corresponding to a change of the length unit. Indicating by $w$ the Weyl rescaling parameter, in 4 dimension we have that the various fields transform as

$$
\left(g_{\mu \nu}, H, \psi_{\alpha}, A_{\mu}\right)=\left(w^{-2} g_{\mu \nu}^{\prime}, w H^{\prime}, w^{3 / 2} \psi_{\alpha}^{\prime}, A_{\mu}^{\prime}\right) .
$$

Indicating collectively the fields with $\Phi$ and the rescaled ones with $\Phi^{\prime}$ a generally covariant action $S$ satisfies the relation

$$
S(\Phi, m)=S\left(\Phi^{\prime}, \frac{m}{w}\right) .
$$

One can easily check this result by considering the free scalar Lagrangian

$$
\sqrt{g}\left(g^{\mu \nu} \partial_{\mu} H \partial_{\nu} H-m^{2} H^{2}\right)
$$

In the case of the matter contribution in eq. (2.23) we make a rescaling with $w=e^{\sigma(\pi)}$ such that $g_{\pi \mu \nu}=w^{-2} \bar{g}_{\mu \nu}, H=w H^{\prime}, \ldots$ and write it as

$$
\sqrt{\bar{g}} \mathcal{L}_{\pi}\left(\bar{g}_{\mu \nu}, H^{\prime}, \psi_{\alpha}^{\prime}, A_{\mu}^{\prime}, m e^{-\sigma(\pi)}\right) .
$$

In these new variables it is evident that all mass parameters in the matter Lagrangian are redshifted with respect to the Planck mass $M_{4} \simeq$ $\sqrt{M_{5}^{3} / k}$. Now the importance of the RS mechanism has become very concrete. We stress once again that the basic reason for this result is that the 4-dimensional Planck mass is dominated by a contribution from the region of low red shift, while the SM lives deep inside a region of high red shift. As a matter of fact the metric $\bar{g}_{\mu \nu}$ that appears in the 4D Einstein term coincides with the induced metric on the Planck brane $g_{0 \mu \nu}$. We will later explain that all these facts corresponds to the localization of the $4 \mathrm{D}$ graviton near the Planck brane.

We want to emphasize that eq. (2.25) implies that only ratios of mass parameters are observable in a theory of gravity, since their overall normalization can be trivially changed by a field redefinition. This is the same situation we have in the SM, where all CP violating phases, but one, transform under field redefinitions and can thus be eliminated. Only the ratio of Planck and weak scales, the hierarchy, is an obervable. Then we can have an alternative view point of our result, where the original scale in the Lagrangian is the weak scale and the Planck mass is a blue shifted derived scale. Consider indeed a rescaling with $w=e^{\sigma(\pi)}$ on our original 
Lagrangian and on our original solution. Now all mass parameters in the starting Lagrangian are of order TeV; for instance $M_{5} \rightarrow M_{5}^{\prime}=M_{5} / w$. Moreover the warp factor is 1 at $y=\pi$ and $e^{\sigma(\pi)}$ at $y=0$. The $4 \mathrm{D}$ Planck mass is now written as

$$
M_{4}^{2}=\frac{\left(M_{5}^{\prime}\right)^{3}}{k^{\prime}}\left(e^{2 k^{\prime} r_{c}^{\prime} \pi}-1\right) .
$$

This picture makes more evident that the the hierarchy is generated by a "dilution" mechanism, like in the ADD scenario. In eq. (2.28), $M_{4}^{2}$ comes out large because of the exponential growth of the "volume" towards the Planck brane.

After the derivation of the effective Lagrangian we can better understand the meaning of the tunings imposed on the boundary tensions. As we will show below, the KK spectrum is quantized in units of the radion VEV $\langle\mu\rangle$. For $E \ll\langle\mu\rangle$ the system can be described by the zero modes $\bar{g}_{\mu \nu}$ and $\mu$. Now, if we add to the brane tensions a perturbation which is small enough not to excite the KK modes we should be able to accurately describe its effects just in terms of the zero modes. Consider then the following perturbations of the tensions parametrized by $\alpha, \beta \ll 1$

$$
\tau_{0}=24 M_{5}^{3} k(1+\alpha) \quad \tau_{\pi}=-24 M_{5}^{3} k(1-\beta) .
$$

At lowest order, the correction to the effective Lagrangian is simply obtained by substituting eq. (2.17) in the terms proportional to $\alpha$ and $\beta$ in the the original Lagrangian

$$
\Delta \mathcal{L}_{4}^{e f f}=-24\left(\frac{M_{5}}{k}\right)^{3} \sqrt{g}\left(\alpha k^{4}+\beta \mu^{4}\right) .
$$

By integrating out the massive KK there arise extra corrections that have either derivatives in them or are of higher order in $\alpha, \beta$. The above equation represents the leading correction to the potential. It is evident that the two tunings of the brane tensions, $\alpha=\beta=0$, correspond to

1 vanishing radion potential

2 vanishing 4-dimensional cosmological constant.

Now, the second requirement is truly necessary since the the cosmological constant is experimentally extremely small $\Lambda_{4}^{4} \lesssim\left(10^{-3} \mathrm{eV}\right)^{4}$. On the other hand, by the first requirement the radion is a massless Brans-Dicke field leading to a new unacceptable long range force. The first tuning is not only useless but experimentally ruled out. The basic RS model must be modified in order to give a mass to the radion while retaining 
the possibility to fine tune the $4 \mathrm{D}$ cosmological contant to zero. In the modified theory there would remain just one tuning.

One can ask if a modifcation of the brane tensions can lead to a realistic radion stabilization. Of course we already know from the derivation of the RS solution that with detuned tensions we would not be able to find a static solution which is also flat from the $4 \mathrm{D}$ viewpoint. But it is useful to study this more quantitatively. Notice that $\beta$ gives rise to a simple quartic potential for $\mu$, so it would seem that the only stationary point is $\mu=0$, which is not interesting. The situation is however slightly more subtle since $\mu$ mixes kinetically with the graviton. In order to easily read the dynamics of $\mu$ it is useful to perform a Weyl rescaling of the metric

$$
\bar{g}_{\mu \nu} \rightarrow \frac{\bar{g}_{\mu \nu}}{1-\frac{\mu^{2}}{k^{2}}}
$$

to go in the Einstein frame in which the gravitational kinetic term is exactly $2\left(M_{5}^{3} / k\right) \sqrt{\bar{g}} \mathcal{R}(\bar{g})$ with no radion contribution. At the same time the radion kinetic term receives a negligibly small modification, while the potential term becomes

$$
\Delta V=-\Delta \mathcal{L}_{4}^{e f f}=24\left(\frac{M}{k}\right)^{3} \sqrt{g} \frac{\alpha k^{4}+\beta \mu^{4}}{\left(1-\frac{\mu^{2}}{k^{2}}\right)^{2}} .
$$

This potential is stationary at $\left(\mu^{2} / k^{2}\right)=-\alpha / \beta$. One can easily check that this stationary point corresponds to a minimum only for $\alpha<0$, $\beta>0$. Around the stationary point, $V$ is negative so that the $4 \mathrm{D}$ metric in turn will be $A d S 4$. It turns out that both the $A d S 4$ curvature $k_{4}$ and the radion mass $m_{\mu}$ scale in the same way $k_{4}^{2} \sim m_{\mu}^{2} \sim-\alpha k^{2}$. Then this solution does not look even approximately like the real world, and a more realistic mechanism of radius stabilization is needed. Before concluding this section we would like to notice that our simple discussion captures and explains in a physically intuitive way the results of refs. $[38,39]$, where the full $5 \mathrm{D}$ equations of motion in the presence of detuned brane tensions were studied. Our approach also clarifies the results of early studies of RS cosmology [40,41], where a puzzling tuning $\rho_{0}=$ $-\rho_{\pi}(\mu / k)^{2}$ between the energy momentum densities at the two different branes was found to be needed. In our particular set up we have $\rho_{0} \equiv k^{4} \alpha$ and $\rho_{\pi} \equiv k^{4} \beta$, showing that the tuning is just the radion stationarity condition in the absence of extra contributions to the potential [42]. 


\subsection{Radius stabilization: Goldberger-Wise mechanism}

As we have already remarked, in the RS model the hierarchy between mass scales at the two boundaries depends exponentially on the proper distance $\pi r_{c}$ between them. A moderate separation $\pi r_{c} / L \sim 40$ between the proper length and the $A d S$ length of the 5th dimension is then enough to obtain a huge hierarchy. This would seem a natural way to explain the hierarchy. The implicit assumption behind this conclusion is that $r_{c}$ is the natural dynamical variable to describe the stabilization of the 5 th dimension, i.e. the radion potential is practically a polynomial in $r_{c}$. However we have so far no solid reason to believe this is the case. As a matter of fact, in the simple example at the end of the previous section, the natural variable was the warp factor $\mu / k$ itself. Now, instead of the proper distance, an observer at the Planck brane could decide to define her/his distance to the $\mathrm{TeV}$ brane through the time $T$ a light signal (or a graviton!) takes to travel to the TeV brane and back. The result would be

$$
T=2 \int_{0}^{\pi} \frac{d y}{\sqrt{g_{00}}}=\frac{2}{k}\left(e^{k r_{c} \pi}-1\right) \sim \frac{2}{\mu}
$$

which is exponentially large as a consequence of time dilation near the $\mathrm{TeV}$ brane. According to this perfectly acceptable definition, the size of the extra dimension is controlled by the weak scale $\mu$ itself. Notice that the potential of the previous section is polynomial in $\mu$. Such hugely different notions for the size of the 5th dimension arise because the global features of the RS space are controlled by curvature. We could have started with a coordinate system in which, according to the above definition of distance, the 5th coordinate is parametrized by

$$
z=\frac{e^{-k r_{c} y}}{k} .
$$

The metric would then have been

$$
d s^{2}=\frac{L^{2}}{z^{2}}\left(\eta_{\mu \nu} d x^{\mu} d x^{\nu}+d z^{2}\right) .
$$

where $L=1 / k$, the $A d S$ radius, represents the $A d S$ curvature length. In these "conformal" coordinates there is no exponential factor, but the locations of the Planck and $\mathrm{TeV}$ branes, respectively $z_{0}$ and $z_{1}$, are very far apart

$$
z_{0}=L \ll L e^{k r_{c} \pi}=z_{1}
$$

In these new coordinates the radion field is simply associated to the position of the second brane: $\mu(x)=1 / z_{1}(x)$. 
To solve the hierarchy problem we must then find a mechanims that stabilizes the second brane at $z_{1} \sim 1 / \mathrm{TeV} \gg z_{0} \sim 1 / M_{P}$. In order to do so, Goldberger and Wise (GW) [35] have proposed a simple model involving a $5 \mathrm{D}$ scalar field $\phi$ with action

$$
\begin{aligned}
S_{\phi} & =\int d^{4} x d z\left\{\sqrt{g}\left[-(\partial \phi)^{2}-m^{2} \phi^{2}\right]+\delta\left(z-z_{0}\right) \sqrt{g_{0}} \mathcal{L}_{0}(\phi)\right. \\
& \left.+\delta\left(z-z_{1}\right) \sqrt{g_{1}} \mathcal{L}_{1}(\phi)\right\}
\end{aligned}
$$

It is assumed that the dynamics of the boundary terms $\mathcal{L}_{0,1}$ is such as to fix the values $\phi\left(z_{0}\right)=\tilde{v}_{0}$ and $\phi\left(z_{1}\right)=\tilde{v}_{1}$. For instance, one can take $\mathcal{L}_{0,1}=-\lambda_{0,1}\left(\phi-v_{0,1}\right)^{2}$ with $\lambda_{0,1} \rightarrow \infty$. The assumption of an infinitely steep boundary potential simplifies the computations but is not essential $[35,36]$. In the vacuum, the field $\phi$ will have a non-trivial bulk profile satisfying the 5D Klein-Gordon equation and interpolating between the two boundary values $v_{0}$ and $v_{1}$. The energy associated to this profile depends on the distance between the two branes, corresponding to a non-vanishing radion potential. Now, solving the coupled equations of motion of gravity plus $\phi$ exactly is in general difficult. To make our task easier we can make the simplifying assumption that $\phi$ only induces a small perturbation of the locally AdS5 Randall-Sundrum metric. Quantitatively this is equivalent to requiring the scalar energy momentum to be a small perturbation of the $5 \mathrm{D}$ cosmological constant

$T_{M N}^{\phi} \sim\left(\partial_{z} \phi\right)^{2}+m^{2} \phi^{2} \sim \max \left(v_{0}^{2}, v_{1}^{2}\right) \times \max \left(k^{2}, m^{2}\right) \ll T_{M N}^{\text {vacuum }} \sim M_{5}^{3} k^{2}$.

Since, in order to generate a big hierarchy we will need $m^{2} \lesssim k^{2}$, the above relation simply implies

$$
v_{0,1}^{2} \ll M_{5}^{3}
$$

(notice that a scalar field in 5D has mass dimension $3 / 2$ ). When this condition holds, $\phi$ is determined at leading order by solving the $\phi$ equations of motion over the unperturbed RS background with Dirichlet boundary conditions $\phi\left(z_{0}\right)=v_{0}$ and $\phi\left(z_{1}\right)=v_{1}$. Because of this second constraint, there are no massless zero modes in the KK tower of $\phi$. As long as the $\phi$ profile is a small perturbation of RS the only light states are those of the unperturbed model: the $4 \mathrm{D}$ graviton and the radion. In this respect the procedure of solving the $\phi$ equations of motion and calculating the action on the solution corresponds to integrating out the tower of massive $\phi \mathrm{KK}$ modes to obtain an effective Lagrangian for the light modes $\bar{g}$ and $\mu$. This is what we will now do. For a field configuration that does not depend on the $4 \mathrm{D}$ coordinates the 5D Klein-Gordon equation 
becomes

$$
\frac{z^{5}}{L^{2}} \partial_{z} \frac{1}{z^{3}} \partial_{z} \phi=m^{2} \phi
$$

and the most general solution is

$$
\phi=A z^{4+\epsilon}+B z^{-\epsilon} \quad \epsilon=\sqrt{4+m^{2} L^{2}}-2 \simeq \frac{m^{2} L^{2}}{4} .
$$

where we are emphasizing that we will be momentarily interested in the limit $\epsilon \sim m^{2} L^{2} / 4 \ll 1$. The boundary conditions fix

$$
A=z_{0}^{\epsilon} \frac{v_{0}-v_{1}\left(z_{0} / z_{1}\right)^{4+\epsilon}}{1-\left(z_{0} / z_{1}\right)^{4+2 \epsilon}} \quad B=z_{1}^{-4-\epsilon} \frac{v_{1}-v_{0}\left(z_{0} / z_{1}\right)^{\epsilon}}{1-\left(z_{0} / z_{1}\right)^{4+2 \epsilon}}
$$

and eq. (2.37) evaluated on the solution yields an effective potential for the radion $\mu$

$$
\begin{aligned}
V(\mu) & =\frac{1}{1-(\mu L)^{4+\epsilon}}\left[(4+\epsilon) \mu^{4}\left(\bar{v}_{1}-\bar{v}_{0}(\mu L)^{\epsilon}\right)^{2}\right. \\
& \left.+\epsilon L^{-4}\left(\bar{v}_{0}-\bar{v}_{1}(\mu L)^{4+\epsilon}\right)^{2}\right] \\
& =L^{-4} F(\mu L)
\end{aligned}
$$

where we have made the substitutions $z_{0}=L, z_{1}=1 / \mu$ and where $\bar{v}_{0,1}=L^{3 / 2} v_{0,1}$ are the boundary VEVs in units of the $A d S$ curvature. For $\mu L \ll 1$ the potential becomes

$$
V=\epsilon \bar{v}_{0}^{2} L^{-4}+\left[(4+2 \epsilon) \mu^{4}\left(\bar{v}_{1}-\bar{v}_{0}\left(\mu / \mu_{0}\right)^{\epsilon}\right)^{2}-\epsilon \bar{v}_{1}^{2} \mu^{4}\right]+\mathcal{O}\left(\mu^{8} L^{4}\right)
$$

which for $\epsilon>0$ is minimized at

$$
\mu L \simeq\left(\frac{\bar{v}_{1}}{\bar{v}_{0}}\right)^{\frac{1}{\epsilon}} .
$$

The hierarchy $\langle\mu\rangle L \sim M_{W} / M_{P}=10^{-17}$ can be naturally obtained for fundamental parameters not much smaller than one (ex. $\bar{v}_{1} / \bar{v}_{0} \sim 1 / 10$ and $\epsilon \sim 1 / 20)$. The hierarchy naturally arises because of the nonanalytic dependence of eq. (2.45) on $\epsilon$. Some comments are now in order.

1 Notice that the relevant part of the potential (the term in square brackets in eq. (2.44)) has the form $\mu^{4} P\left(\mu^{\epsilon}\right)$. This is basically a quartic potential modulated by a slow evolution of the effective coupling $\lambda \equiv P\left(\mu^{\epsilon}\right)$. Notice indeed that for $\epsilon \ll 1$ one has $\mu^{\epsilon} \sim$ $1+\epsilon \ln \mu+\ldots$, so that the dependence on $\mu$ is reminiscent of a slow 
RG evolution. Stability of the potential at large $\mu$ corresponds to $\lim _{\mu \rightarrow \infty} P(\mu)>0$. Because of the slow evolution of $P$, a minimum of the potential will exist very close to the point where $P$ crosses zero to become negative, see eqs. $(2.44,2.45)$. These properties are in full analogy with the Coleman-Weinberg (CW) [37] mechanism of dimensional transmutation. There, quantum corrections to the effective potential cause the quartic coupling to turn negative at some scale. Since this happens through the slow logarithmic RG evolution, broadly different mass scales can arise, making the CW mechanism very interesting to explain the weak to Planck scale hierarchy. It seems that the GW mechanism works qualitatively in the same way.

2 Expanding at second order around the minimum and using the unperturbed kinetic Lagrangian we find the radion mass

$$
m_{\mu}^{2} \simeq \frac{2}{3} \bar{v}_{1}^{2} \epsilon^{3 / 2}\langle\mu\rangle^{2} \ll\langle\mu\rangle^{2}
$$

indicating that in the model at hand the radion is much lighter than the other KK resonances and potentially more interesting for collider phenomenology.

3 The potential at the minimum is dominated by the fist term in eq. (2.44): $V_{\min } \simeq \epsilon \bar{v}_{0}^{2} L^{-4} \sim M_{4}^{4}$, far too large! However we can now go back and slightly modify the brane tensions as we did in the previous section. For small $\alpha$ and $\beta$, at leading order this amounts to adding the contribution in eq. (2.30) to eq. (2.44). Around the minimum of the GW potential $\mu$ is massive so that $\beta \neq 0$ causes only a small shift in $\langle\mu\rangle$. We no longer need to tune $\beta=0$ after the radius is stabilized. Moreover both $\alpha$ and $\beta$ cause a shift in the potential at the minimum. By properly choosing one combination of them we can cancel the potential at the minimum. This is of course a fine tuning, but it is just the cosmological constant problem, which we never promised to solve. After radius stabilization the only fine tuning left in the model is the one associated to the vanishing cosmological constant.

4 The minimal GW potential gives rise to a stable minimum only for $\epsilon \simeq m^{2} L / 4>0$. However one could also obtain stable minima for $\epsilon<0$ for a proper range of the detuning parameter $\beta$.

Concerning the last remark one may worry that $\epsilon \sim m^{2}<0$ will lead to instabilities in the bulk. If $\left|m^{2}\right|$ is small enough, however, no 
instability arises. Concerning this result it is instructive to consider the following exercises.

- Show that no instability is generated for $m^{2}>-4 k^{2}+$ (small) (hint: study the KK spectrum for the excitation $\Delta \phi$ around the GW background remembering that $\Delta \phi=0$ at the boundaries).

- Study the same problem with a flat 5th dimension.

\subsection{Kaluza-Klein spectrum}

In this section we shall study the KK spectrum of the gravitational field. As for the flat case we studied earlier, it is convenient to use the $5 \mathrm{D}$ gauge freedom to eliminate as many degrees of freedom from $g_{\mu 5}$ and $g_{55}$ as possible. It is then easy to check (for instance by working at the linearized level) that without loss of generality we can use the following parametrization

$$
d s^{2}=e^{-2 k|y| r_{c}-2 f(x) e^{2 k|y| r_{c}}}\left(\hat{g}_{\mu \nu}(x, y) d x^{\mu} d x^{\nu}\right)+\left(1+2 f(x) e^{2 k|y| r_{c}}\right)^{2} d y^{2}
$$

Because of the orbifold projection we can consistently set $g_{\mu 5} \equiv 0$. On the other hand for $g_{55}$ we can eliminate all the modes but one, the radion. This one mode remaining in $g_{55}$ can however be parametrized at our convenience, and the choice in eq. (2.47) as an advantage that we will now explain. Since we are mostly interested in the $J=2$ modes, consider first substituting eq. (2.47) with $f(x)=0$ in the RS action. After changing the 5th coordinate to $z=L e^{-k|y| r_{c}}$, one finds

$$
S(g)=4 M_{5}^{3} L^{3} \int_{L}^{z_{1}} \sqrt{\hat{g}} \frac{d z}{z^{3}}\left[\mathcal{R}_{4}(\hat{g})+\frac{1}{4}\left(\left(\partial_{z} \hat{g}^{\mu \nu}\right)\left(\partial_{z} \hat{g}_{\mu \nu}\right)+\left(\hat{g}^{\mu \nu} \partial_{z} \hat{g}_{\mu \nu}\right)^{2}\right)\right] .
$$

Notice that all terms with no derivative acting on $\hat{g}_{\mu \nu}(x, y(z))$ have judiciously cancelled out when expanding around the RS solution. We already know that it should have been this way: any constant $\hat{g}_{\mu \nu}$ solves the equations of motion, so that the action for $\hat{g}_{\mu \nu}$ must involve only gradient terms. When $f \neq 0$ we find an extra contribution to be added to eq. (2.48) and starting at quadratic order in $f$

$$
\Delta S=-12 \frac{M_{5}^{3}}{L}\left(z_{1}^{2}-L^{2}\right) \partial_{\mu} f \partial^{\mu} f+O\left(f^{3}, f^{2} h_{\mu \nu}, \ldots\right)
$$

where $h_{\mu \nu}=\hat{g}_{\mu \nu}-\eta_{\mu \nu}$. Notice that there is no kinetic mixing between $f$ and the metric $h_{\mu \nu}$. In other words, the graviton is automatically in the Einstein frame. This is because of our specific parametrization of the 
scalar mode in eq. (2.47). This should be contrasted to the parametrization in eq. (2.17) which lead to a small $O\left(\mu^{2} L^{2}\right)$ mixing between radion and graviton. Anyway, neglecting $O(\mu L)$, terms and with the identification (cfr. eqs. $(2.17,2.19))$

$$
\mu(x)=k e^{-k \pi r_{c}-f(x) e^{2 k \pi r_{c}}}=k e^{-\sigma(\pi, x)}
$$

eq. (2.49) reproduces the radion kinetic term of eq. (2.18).

Let us now focus on the $J=2$ modes. Expanding eq. (2.48) at quadratic order in $h_{\mu \nu}=\hat{g}_{\mu \nu}-\eta_{\mu \nu}$ we find

$$
M_{5}^{3} L^{3} \int_{L}^{z_{1}} \frac{d z}{z^{3}}\left(h_{\mu \nu} K^{\mu \nu \rho \sigma} h_{\rho \sigma}-h^{\mu \nu} \partial_{z}^{2} h_{\mu \nu}+h_{\mu}^{\mu} \partial_{z}^{2} h_{\nu}^{\nu}\right)
$$

where $K^{\mu \nu \rho \sigma}$ is the 4-dimensional $J=2$ kinetic operator shown in the first line of eq. (1.39). Notice that the mass operator has the Fierz-Pauli form. From eq. (2.51) the equation for the eigenmodes $\psi_{n}(z)$ is then simply

$$
-z^{3} \partial_{z} \frac{1}{z^{3}} \partial \psi_{n}(z)=m_{n}^{2} \psi_{n}(z)
$$

with boundary conditions

$$
\left.\partial_{z} \psi_{n}\right|_{z=L}=\left.\partial_{z} \psi_{n}\right|_{z=z_{1}}=0
$$

as $h_{\mu \nu}$ is even under the orbifold parity and its action does not contain boundary terms.

For $m_{n} \neq 0$ the general solution to eq. (2.52) is written in terms of Bessel functions

$$
\psi_{n}(z)=\frac{z^{2}}{L^{2}}\left[J_{2}\left(m_{n} z\right)+b_{n} Y_{2}\left(z m_{n}\right)\right]
$$

and the boundary conditions enforced by using the identity

$$
\partial_{z} \psi_{n} \propto z^{2}\left[J_{1}\left(m_{n} z\right)+b_{n} Y_{1}\left(z m_{n}\right)\right] .
$$

We then find

$$
b_{n}=\frac{J_{1}\left(m_{n} L\right)}{Y_{1}\left(m_{n} L\right)}
$$

while the eigenvalue equation is simply

$$
J_{1}\left(m_{n} L\right) Y_{1}\left(m_{n} z_{1}\right)-Y_{1}\left(m_{n} L\right) J_{1}\left(m_{n} z_{1}\right)=0 .
$$

In order to focus on the phenomenologically interesting "light" modes, satisfying $m_{n} L \ll 1$, we need the asymptotic behaviour of the Bessel functions

$$
x \ll 1 \quad \rightarrow \quad\left\{\begin{array}{l}
J_{2}(x)=\frac{x^{2}}{8}+O\left(x^{4}\right) \\
Y_{2}(x)=-\frac{4}{\pi x^{2}}-\frac{1}{\pi}+O\left(x^{2}\right)
\end{array}\right.
$$




$$
x \gg 1 \quad \rightarrow \quad\left\{\begin{array}{l}
J_{2}(x)=\sqrt{\frac{2}{\pi x}} \cos \left(x-\frac{5}{4} \pi\right) \\
Y_{2}(x)=-\sqrt{\frac{2}{\pi x}} \sin \left(x-\frac{5}{4} \pi\right)
\end{array}\right.
$$

In the limit $m_{n} L \ll 1$ eqs. (2.56) and (2.57) reduce respectively to

$$
b_{n} \simeq\left(m_{n} L\right)^{2} \pi / 4 \ll 1
$$

and

$$
J_{1}\left(m_{n} z_{1}\right)=0
$$

The solutions to the last equation are quantized in units of $1 / z_{1} \equiv\langle\mu\rangle$

$$
m_{n}=c_{n} \frac{1}{z_{1}} \simeq\left(n+\frac{1}{4}\right) \frac{\pi}{z_{1}},
$$

where the last identity is valid asymptotically for $n \gg 1$ (cfr. eq. (2.58)) but works very well already for $n=1: c_{1} \simeq 1.21 \pi$. Notice that because $b_{n} \ll 1, Y_{2}$ makes a negligible contribution to $\psi_{n}$ in the region $m_{n} z \gg$ 1 where $\psi_{n}$ oscillates. In the region $z m_{n} \ll 1$, the $Y_{2}$ contribution is relatively important (in fact dominant), but this region contributes negligibly to the normalization of the eigenfunctions.

Indeed, by eq. (2.51) the norm of the modes is

$$
\begin{aligned}
\left\|\psi_{n}\right\|^{2} & =\int_{z_{0}=L}^{z_{1}} \frac{2 z d z}{L^{2}}\left[J_{2}\left(m_{n} z\right)+b_{n} Y_{2}\left(m_{n} z\right)\right]^{2} \simeq \int_{0}^{z_{1}} \frac{z d z}{L^{2}}\left[J_{2}\left(m_{n} z\right)\right]^{2} \\
& =\left(\frac{z_{1}}{L}\right)^{2} J_{2}\left(m_{n} z_{1}\right)^{2} .
\end{aligned}
$$

Neglecting contributions of relative size $O\left(L^{2} / z_{1}^{2}\right) \sim m_{\text {weak }}^{2} / M_{P}^{2}$, we have approximated $\left\|\psi_{n}\right\|$ by its value in the limit $z_{0}=0$, in which the Planck brane has an infinite proper distance from the $\mathrm{TeV}$ brane (and an infinite relative blueshift, see eq. (2.35)). $\left\|\psi_{n}\right\|$ is dominated by the region $1 / m_{n}<z<z_{1}$, where the Bessel functions have an oscillatory behaviour. A mode with mass $m_{n}$ is not very sensitive to the region $z<1 / m_{n}$. In particular the modes remain normalizable and the spectrum discrete even for $z_{0} \rightarrow 0$. Notice finally that the orthonormal modes $\hat{\psi}_{n}(z) \equiv \psi_{n}(z) /\left\|\psi_{n}\right\|$ satisfy $\hat{\psi}_{n}\left(z_{1}\right)=\operatorname{sgn}\left(J_{2}\left(m_{n} z_{1}\right)\right) z_{1} / L$, so that all modes couple with equal strength to the fields on the SM brane.

The massless mode, see eq. (2.52), simply corresponds to a constant $\psi_{0}=1$ with norm

$$
\left\|\psi_{0}\right\|=L^{2} \int_{z_{0}=L}^{z_{1}} \frac{2 d z}{z^{3}}=\left(\frac{1}{L^{2}}-\frac{1}{z_{1}^{2}}\right)
$$


which is dominated at small $z$. Unlike the massive modes, $\psi_{0}$ is localized at the Planck brane: $\left\|\psi_{0}\right\|$ diverges for $z_{0} \rightarrow 0$ but remains finite for $z_{1} \rightarrow \infty$. From eq. (2.48) we have that the $4 \mathrm{D}$ Planck mass is $M_{4}^{2}=M_{5}^{3} L^{3}\left\|\psi_{0}\right\|^{2}=M_{5}^{3} L\left[1-\left(L / z_{1}\right)^{2}\right]$, which is the result we obtained previously. Notice also that for $z_{0} \rightarrow 0$ the $4 \mathrm{D}$ Planck mass diverges, corresponding to the zero mode graviton being localized infinitely far away from the $\mathrm{TeV}$ brane.

Using the above results, but working with the canonically normalized KK gravitons $\bar{h}_{\mu \nu}^{(n)}$ and radion $\bar{f}$ the coupling to the TeV brane energy momentum tensor is written as

$$
\mathcal{L}_{i n t}=-\frac{1}{2}\left\{\frac{\bar{h}_{\mu \nu}^{(0)}}{M_{4}}+\sum_{n \geq 1} \frac{\bar{h}_{\mu \nu}^{(n)}}{\Lambda}\right\} T^{\mu \nu}+\frac{\bar{f}}{\sqrt{24} \Lambda} T_{\mu}^{\mu}
$$

where $\Lambda \equiv\left(M_{5} L\right)^{3 / 2} / z_{1}$. Eq. (2.64) is the basic equation to study the collider implications of the RS model [43]. The interactions and spectrum of the $J=2$ modes are fully described by two parameters, $\Lambda$ and $\langle\mu\rangle=1 / z_{1}$. For radion phenomenology two extra parameters are needed, one is the radion mass, which depends on the stabilization mechanism, the other is a radion-Higgs mixing parameter $\xi$ [24]. Basically the parameter $\xi$ accounts for the fact that the energy momentum tensor for a scalar $H$ is defined up to an "improvement term" $\Delta T_{\mu \nu}=\xi\left(\partial_{\mu} \partial_{\nu}-\eta_{\mu \nu} \partial^{2}\right) H^{2}$. A non zero $\xi$ induces a kinetic mixing between radion and Higgs after electroweak symmetry breaking.

A little exercise one can do with the interaction Lagrangian in eq. (2.64) concerns the validity of perturbation theory. A simple quantity to calculate (estimate) is the decay width into $\mathrm{SM}$ particles (living at the $\mathrm{TeV}$ boundary). For the $n$-th mode we find

$$
\Gamma_{(n)} \sim \frac{m_{n}^{3}}{8 \pi \Lambda^{2}} .
$$

Notice that $\Gamma_{(n)}$ grows with $n$, so that for $n$ large enough the nearby resonances will start overlapping. When this takes place, by definition, perturbation theory breaks down: quantum corrections to the spectrum (the widths) make the very concept of individual KK resonances useless. Using the above equations we find that $\Gamma_{(n)}<m_{n+1}-m_{n}$ is satisfied for masses below

$$
m_{n}<M_{5} \frac{L}{z_{1}}
$$

This shows that the UV cut-off for physics at the TeV scale is just the redshifted Planck mass. Of course by starting off in the primed Weyl frame, discussed above eq. (2.28), this is the obvious result. 


\subsection{Strong coupling puzzle}

At the end of the previous section we have shown by a simple argument that the RS model becomes strongly coupled at a fairly low scale $M^{\prime}{ }_{5}=$ $M_{5} L / z_{1}$. If we we want to explain the hierarchy by the ratio $L / z_{1}$, then $M^{\prime}{ }_{5} \sim \mathrm{TeV}$ very much like in the ADD scenario. Although such a lowcut off limits predictivity, as long as $M_{5}^{\prime}$ is somewhat bigger than the mass of the lightest KK, some control is retained: roughly a number of modes $\sim M^{\prime}{ }_{5} z_{1}=M_{5} L$ remains weakly coupled. From the conceptual viewpoint, however, the presence of this low cut-off can be confusing, when not properly interpreted. One basic puzzle is that $M^{\prime}{ }_{5}$ depends on the location of the TeV brane. Moreover as $z_{1} \rightarrow \infty$ and half of $A d S 5$ is recovered, $M^{\prime}{ }_{5}$ goes to zero, as if there was no energy range where gravity on $A d S$ makes sense as an effective field theory. The origin of this puzzle is that we are working on a curved space where particle propagation involves large or possibly infinite momentum blueshift. As we will explain, since the puzzle arises when considering the global aspect of our spacetime, a proper resolution cannot do without accounting for the locality of the interactions.

It is instructive to go back and consider the motion of a particle in $A d S$ space. The equations for the geodetic in conformal coordinates are

$$
\ddot{x}^{\mu}-2 \frac{\dot{x}^{\mu} \dot{z}}{z}=0 \quad \ddot{z}-\frac{\dot{z}^{2}}{z}+\frac{\dot{x}^{\mu} \dot{x}^{\nu} \eta_{\mu \nu}}{z}=0
$$

where by the dot we indicate the derivative with respect to the affine parameter $\lambda$. Let us focus on the massless case. As $\lambda$ is arbitrary, we can choose it such that $d x^{M} / d \lambda$ coincides with the 5 -momentum $P^{M}$. By this choice we have, in particular, $d / d \lambda=P^{5} d / d z$ and by using the zero mass condition $P^{M} P^{N} g_{M N}=0$ we can write eq. (2.67) and its solution as

$$
z \frac{d P^{M}}{d z}=2 P^{M} \quad \rightarrow \quad P^{M}=\left(\frac{z^{2}}{L^{2}}\right) \bar{P}^{M} .
$$

This is the momentum in the AdS coordinates, but a more physical quantity is the momentum $P_{\text {phys }}^{M}$ seen in the frame of a free falling observer at rest at $z: P_{\text {phys }}^{M}=P^{M}(L / z)=\bar{P}^{M}(z / L)$. Viewing the particle as a wave, the increase in momentum is simply due to the homogeneous contraction $L / z$ of all lengths when moving down the $A d S$ throat, see Fig. (9). Moving to larger $z$ is like going backward in time in Friedmann-RobertsonWalker (FRW) cosmology. This analogy is not accidental as both $A d S$ and FRW have conformally flat metrics. Now, consider two particles starting with momenta $\bar{P}_{1}^{M}$ and $\bar{P}_{2}^{M}$ at the Planck brane. If they collide after falling at a bulk position $z$, the center of mass energy of the collision 
will be $s(z)=-2 P_{1}^{M} P_{2}^{N} g_{M N}=-2 \bar{P}_{1}^{M} \bar{P}_{2}^{N} \eta_{M N}(z / L)^{2}=s(0)(z / L)^{2}$. Then by starting by a sub-Planckian energy $\sqrt{s}(0)<M_{5}$ we can produce super-Planckian collisions if $z$ is large enough. Of course this also means that a Planck brane observer must wait a relatively long time $T>z$ in order to oberve this collision. In a moment we will see that this time delay is the central point to discuss "strong coupling" at the quantum level. Notice also that in the case of the compact RS model $z$ is bounded by $z_{1}$, so that for $\sqrt{s(0)}<M_{5} L / z_{1}$ the Planck mass is never exceeded in the bulk. Once again we have found that the critical energy corresponds to the redshifted Planck mass $M_{5}^{\prime}$.

Consider now the limit $z_{1} \rightarrow \infty$. At $z=\infty$ the metric of $4 \mathrm{D}$ slices vanishes, similarly to what happens to $g_{00}$ in the Schwarzschild solution in GR. Also, at the point $z=\infty$ there is a horizon, the $A d S$ horizon. In fact a particle falling from the Planck brane takes an infinite Planck brane time to reach $z=\infty$, but the proper time experienced by the particle is finite $\tau=\pi L / 2$. This is completely analogous to the Schwarzschild case. The model so defined is named RSII [44], so that the model with two branes we have considered so far is named RSI. If we assume that the $\mathrm{SM}$ is instead localized at the Planck brane (and thus give up explaining the hierarchy by redshifts) the RSII model represents an "alternative to compactification". This is because, although the 5th dimension is non-compact, there is still a normalizable 4-dimensional graviton, see eq. (2.63), dominating the IR behaviour of gravity. Eq. (2.63) should be contrasted to the flat case, in which $\left\|\psi_{0}\right\|$ grows with the radius $R$, so that the zero mode decouples in the infinite volume limit.

The RSII model seems a very interesting way to view $4 \mathrm{D}$ gravity. In this model however a particle falling from the Planck brane can undergo virtually infinite Redshift before colliding. Then if we blindly applied the above definition of UV cut-off we would conclude that RSII is not a viable effective field theory! And we would be wrong. The point is that the notion of "which energy is Planckian" must be a local one. As we have already stated, a Planck brane observer, while working with initial states that have $\sqrt{s} \ll M_{5}$, must set up an experiment that probes deep into the $5 \mathrm{D}$ bulk in order to see quantum gravity effects. An instructive way to understand how things work is to consider a scattering process induced by graviton exchange among particles localized on the Planck brane. Consider first a $t$-channel process at fixed angle, for which the exchanged graviton has virtuality $Q=\sqrt{-t} \sim \sqrt{s}$. A particle with virtuality $Q$ can exist only for a time $\sim 1 / Q$, so that by causality it probes the bulk no further than $z_{Q} \sim 1 / Q$. The virtual momentum is 


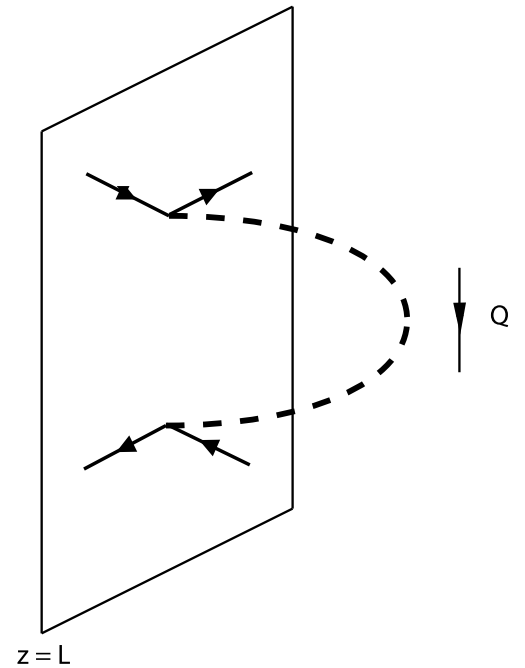

Figure 10. Amplitude for the scattering of fermions localized on the Planck brane via $t$-channel $5 D$ graviton exchange.

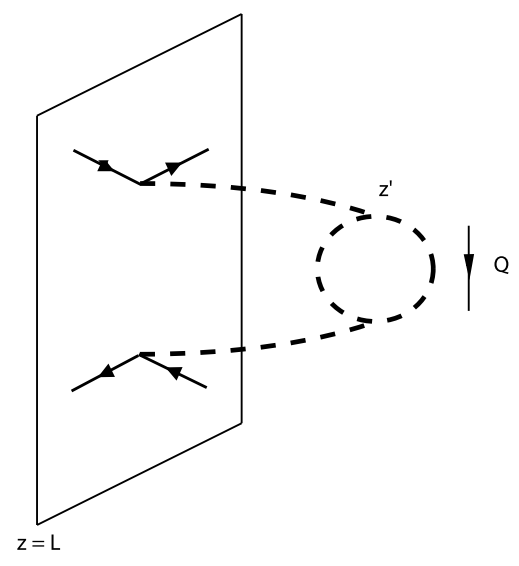

Figure 11. Leading quantum gravity correction to the process of Fig. (10).

then only blueshifted to

$$
Q(z)=Q \frac{z}{L} \lesssim Q \frac{z_{Q}}{L} \sim \frac{1}{L}
$$

so that perturbativity of the process is maintained as long as $1 / L \ll M_{5}$. But this was our original requirement in order to trust the RS solution. By the above simple argument we expect the leading gravitational loop corrections to the above process to be controlled by $Q\left(z_{Q}\right) / M_{5}=$ $1 /\left(M_{5} L\right)$ independent of the scale of the kinematical parameters $s$ and $t=Q^{2}$. This result is remarkable: the leading gravitational corrections are scale invariant. This does not happen by chance and is related to the possibility to interpret the RSI and RSII models according to the $A d S / C F T$ correspondence $[46,47,36]$. As shown in Fig. (11), we can make our arguments slightly more concrete by considering the 1-loop correction to our $t$-channel process. The crucial remark [47] is that the brane to bulk propagator (working in momentum space along the brane and in position space along the 5 th dimension) behaves like

$$
G\left(z=z_{0}, z^{\prime}, Q\right) \propto e^{-Q\left(z^{\prime}-z_{0}\right)}
$$

while the local loop expansion parameter is $\left[Q\left(z^{\prime}\right)\right]^{3} / M_{5}^{3}$. Taking the brane to bulk propagator into account, the 1-loop correction diagram 
will be of order

$$
e^{-Q\left(z^{\prime}-L\right)}\left(\frac{Q z^{\prime}}{M_{5} L}\right)^{3}<\frac{1}{\left(M_{5} L\right)^{3}}
$$

consistent with our previous result. Notice that both the above inequality and the previous argument apply only for $Q<1 / L$. When $Q>1 / L$ curvature is unimportant (the relevant length scale $1 / Q$ is short) so that the loop expansion parameter is just that of flat space $\sim Q^{3} / M_{5}^{3}$. We conclude that a Planck brane observer studying $t$-channel graviton exchange sees quantum gravity becoming important at the most obvious scale: $M_{5}$. By the same argument, we would also deduce that an observer sitting on a probe brane at position $z$ sees gravity becoming strong at a local scale $M_{5}(z)=M_{5} z / L$.

The situation for $s$-channel processes is somewhat different. Here with enough energy resolution one could produce, even on the Planck brane, individual KK-modes and measure their widths, being thus able to decide whether there is strong dynamics. The required energy resolution $\Delta E \lesssim 1 / z_{1}$, corresponds, by the indetermination principle, to a time scale $T>z_{1}$ during which a signal can travel from the Planck to TeV brane. To be more definite consider the annihilation process $\lambda \bar{\lambda} \rightarrow \chi \bar{\chi}$ for fermions living on the Planck brane. The $s$-channel amplitude is

$$
\begin{aligned}
A(s)= & \frac{1}{4} T^{\mu \nu}(\text { in }) T^{\rho \sigma}(\text { out })\left\langle h_{\mu \nu} h_{\rho \sigma}\right\rangle \\
= & \frac{1}{4} T^{\mu \nu}(\text { in }) T^{\rho \sigma}(\text { out }) \\
& \sum_{n} \frac{\left|\hat{\psi}^{(n)}(z=L)\right|^{2}}{M_{5}^{3} L} \frac{\Pi_{\mu \nu \rho \sigma}\left(m_{n}\right)(s)}{s-m_{n}^{2}+i m_{n} \Gamma_{n}}
\end{aligned}
$$

where $\Pi\left(m_{n}\right)$ is the $J=2$ projection operator defined in eq. (1.43) and where for simplicity we have neglected the radion contribution. The wave function at the Planck brane is $\left|\hat{\psi}^{(n)}(z=L)\right|^{2} \sim m_{n}\left(L^{2} / z_{1}\right)$ for the massive modes, but for the zero mode we have $\left|\hat{\psi}^{(0)}(z=L)\right|^{2} \sim 1$. The lighter massive modes, being localized away from the Planck brane, couple much more weakly than the zero mode. In this respect, the production of these modes is very suppressed. However even with this tiny coupling one could in principle study the production of individual resonances and measure their width. In fact we do not want to stress to much the wave function suppression, as it is a specific feature of the graviton KK. For instance, in the case of a bulk vector this suppression is practically absent [45]. The point we want to stress here concerns instead the energy resolution of the experiment. In order to proceed we need the explicit expression of the brane to brane propagator as a sum 
over massive KK , 4D graviton and radion contributions

$$
M_{5}^{3}\left\langle h_{\mu \nu} h_{\rho \sigma}\right\rangle=A(s) \Pi_{\mu \nu \rho \sigma}^{m \neq 0}+\frac{z_{1}^{2}}{z_{1}^{2}-L^{2}} \frac{\Pi_{\mu \nu \rho \sigma}^{m=0}}{s L}+\frac{L^{2}}{z_{1}^{2}-L^{2}} \frac{\eta_{\mu \nu} \eta_{\rho \sigma}}{6 s L}
$$

where

$$
\Pi_{\mu \nu \rho \sigma}^{m \neq 0}=\frac{1}{2}\left(\eta_{\mu \rho} \eta_{\sigma \nu}+\eta_{\mu \sigma} \eta_{\rho \nu}\right)-\frac{1}{3} \eta_{\mu \nu} \eta_{\rho \sigma}
$$

is the massive spin 2 projector of eq. (1.43) after eliminating the irrelevant longitudinal parts, while $\Pi_{\mu \nu \rho \sigma}^{m=0}$ is the massless projector given in eq. (1.46). Notice that the radion contribution, the third term, is suppressed by a power $L^{2} / z_{1}^{2}$. The massive $\mathrm{KK}$ amplitude is

$$
\begin{aligned}
A(s) & =\left\{\frac{1}{2 \sqrt{s}} \frac{Y_{1}\left(\sqrt{s} z_{1}\right) J_{2}(\sqrt{s} L)-J_{1}\left(\sqrt{s} z_{1}\right) Y_{2}(\sqrt{s} L)}{Y_{1}\left(\sqrt{s} z_{1}\right) J_{1}(\sqrt{s} L)-J_{1}\left(\sqrt{s} z_{1}\right) Y_{1}(\sqrt{s} L)}-\frac{1}{s L} \frac{z_{1}^{2}}{z_{1}^{2}-L^{2}}\right\} \\
& \equiv \frac{F(s)}{2 \sqrt{s}}-\frac{1}{s L} \frac{z_{1}^{2}}{z_{1}^{2}-L^{2}} .
\end{aligned}
$$

Notice that $A(s)$, and $F(s)$ have poles on the real positive axis in correspondence with the massive KK masses. However $A(s)$ does not have a pole at $s=0$, while $F(s)$ does. For large $z_{1}$, on the positive real $s$ axis $A(s)$ is a complicated oscillating function with narrowly spaced poles. However when $s$ is continued into the complex plane all this structure gives way to the much simpler euclidean behaviour. By giving $\sqrt{s}$ a positive finite imaginary part $\sqrt{s} \equiv q_{R}+i q_{I}$ and by using the asymptotic expansion for Bessel functions we find

$$
\begin{aligned}
F(s) & =\frac{J_{2}(\sqrt{s} L)+i Y_{2}(\sqrt{s} L)}{J_{1}(\sqrt{s} L)+i Y_{1}(\sqrt{s} L)}\left(1+O\left(e^{-2 q_{I} z_{1}}\right)\right) \\
& =\frac{H_{2}^{(1)}(\sqrt{s} L)}{H_{1}^{(1)}(\sqrt{s} L)}\left(1+O\left(e^{-2 q_{I} z_{1}}\right)\right)
\end{aligned}
$$

where we employed the definition of the Hankel functions. Substituting this result into eq. (2.75) we find, up to $O\left(e^{-2 q_{I} z_{1}}\right)$ terms

$$
\left\langle h_{\mu \nu} h_{\rho \sigma}\right\rangle\left(\sqrt{s}=q_{R}+i q_{I}\right)=-\frac{H_{2}^{(1)}(\sqrt{s} L)}{2 \sqrt{s} H_{1}^{(1)}(\sqrt{s} L)} \Pi_{\mu \nu \rho \sigma}^{m \neq 0}-\frac{\eta_{\mu \nu} \eta_{\rho \sigma}}{6 s L} .
$$

The important point about this result is that all the dependence on powers of $z_{1}$ has disappeared. Now, the point is that if we use initial states that have an energy spread $(\Delta s) / \sqrt{s}>>1 / z_{1}$ (i.e. smearing this amplitude with a wave function spread over $\Delta s$ ) is practically equivalent to considering the amplitude at a complex point with $\operatorname{Im}(\sqrt{s}) \sim i \Delta s / \sqrt{s}$. 
For such spread states all the physics of the KK-modes, including their detailed strong dynamics is exponentially suppressed, and thus practically inaccessible. This result is quite analogous to the well known example of $e^{+} e^{-} \rightarrow$ hadrons. In that case, the behaviour of the cross section as a function of the energy can be very complicated by the presence of the resonances, indicating that a perturbative QCD computation is not adequate. For instance this is the case near the bottom quark threshold. However by averaging the cross section over $\Delta s / \sqrt{s}>>\Lambda_{Q C D}$ one obtains an observable which can be reliably computed in perturbation theory in terms of the production of quarks and gluons. This is the so-called parton-hadron duality.

To conclude we want to apply eq. (2.77) in the fully euclidean region $\sqrt{s}=i q$ to compute the gravitational potential induced on the Planck brane by a source on it. Notice that this is the exact propagator for RSII, while for RSI it applies only for $q>1 / z_{1}$. We are interested in the long distance behaviour $q L \ll 1$, so we use the Bessel function expansion at small argument.

We find

$$
\left\langle h_{\mu \nu} h_{\rho \sigma}\right\rangle(\sqrt{s}=+i q)=\frac{1}{M_{5}^{3} L}\left\{\frac{\Pi_{\mu \nu \rho \sigma}^{m=0}}{q^{2}}+\Pi_{\mu \nu \rho \sigma}^{m \neq 0} \frac{L^{2}}{2} \ln q^{2}+O\left(q^{2}\right)\right\}
$$

so that the Newtonian potential induced by a body of mass $m$ is

$$
V(r)=\frac{G_{N} m}{r}\left(1+\frac{2 L^{2}}{3 r^{2}}+\ldots\right) .
$$

This formula applies in the long distance regime $r \gg L$. A good exercise is to check that at a distance $r \ll L$, the potential goes back to the 5D behaviour $V \sim 1 / r^{2}$. Then RSII can be a viable alternative to compactification for $L \lesssim 100 \mu \mathrm{m}$. 



\section{References}

[1] J. Polchinski, "TASI lectures on D-branes," arXiv:hep-th/9611050.

[2] N. Arkani-Hamed, S. Dimopoulos and G. Dvali, Phys. Lett. B429, 263 (1998) [hep-ph/9803315].

[3] L. Randall and R. Sundrum, Phys. Rev. Lett. 83 (1999) 3370 [hep-ph/9905221].

[4] see for instance R. Rajaraman, Solitons and instantons : an introduction to solitons and instantons in quantum field theory, North-Holland, 1982.

[5] V. A. Rubakov and M. E. Shaposhnikov, Phys. Lett. B 125 (1983) 136.

[6] G. R. Dvali and M. A. Shifman, Phys. Lett. B 396, 64 (1997) [Erratum-ibid. B 407, 452 (1997)] [arXiv:hep-th/9612128].

[7] R. Sundrum, Phys. Rev. D 59, 085009 (1999) [arXiv:hep-ph/9805471].

[8] I. Low and A. V. Manohar, Phys. Rev. Lett. 88, 101602 (2002) [arXiv:hepth/0110285].

[9] D. B. Kaplan, arXiv:nucl-th/9506035; A. V. Manohar, arXiv:hep-ph/9606222; I. Z. Rothstein, arXiv:hep-ph/0308266.

[10] J. F. Donoghue, Phys. Rev. D 50, 3874 (1994) [arXiv:gr-qc/9405057].

[11] J. Polchinski, Nucl. Phys. B 231269 (1984).

[12] S. Weinberg, Phys. Lett. B 91, 51 (1980).

[13] T. Kaluza, Sitzungsber. Preuss Akad. Wiss. Berlin (Math. Phys.) 1921, 966 (1921); O. Klein, Z. Phys. 37, 895 (1926).

[14] M. Fierz and W. Pauli, Proc. Roy. Soc. Lond. A 173 (1939) 211.

[15] G. F. Giudice, R. Rattazzi and J. D. Wells, Nucl. Phys. B 544, 3 (1999); E. A. Mirabelli, M. Perelstein and M. E. Peskin, Phys. Rev. Lett. 82, 2236 (1999);T. Han, J. D. Lykken and R. J. Zhang, Phys. Rev. D 59, 105006 (1999); J. L. Hewett, Phys. Rev. Lett. 82, 4765 (1999).

[16] I. Antoniadis, N. Arkani-Hamed, S. Dimopoulos and G. Dvali, Phys. Lett. B436, 257 (1998) [hep-ph/9804398]. 
[17] see for instance G. Aldazabal, S. Franco, L. E. Ibanez, R. Rabadan and A. M. Uranga, JHEP 0102, 047 (2001) [arXiv:hep-ph/0011132];G. Aldazabal, L. E. Ibanez, F. Quevedo and A. M. Uranga, JHEP 0008, 002 (2000) [arXiv:hepth/0005067]; I. Antoniadis, arXiv:hep-th/0102202.

[18] N. Arkani-Hamed, S. Dimopoulos and J. March-Russell, Phys. Rev. D 63, $064020(2001)$

[19] N. Arkani-Hamed, L. J. Hall, D. R. Smith and N. Weiner, Phys. Rev. D 62, 105002 (2000) [arXiv:hep-ph/9912453].

[20] N. Arkani-Hamed, S. Dimopoulos and G. Dvali, Phys. Rev. D59, 086004 (1999) [hep-ph/9807344].

[21] E. G. Adelberger [EOT-WASH Group Collaboration], arXiv:hep-ex/0202008.

[22] J. Chiaverini, S. J. Smullin, A. A. Geraci, D. M. Weld and A. Kapitulnik, Phys. Rev. Lett. 90, 151101 (2003)

[23] J. C. Long, H. W. Chan, A. B. Churnside, E. A. Gulbis, M. C. Varney and J. C. Price, Nature 421 (2003) 922.

[24] G. F. Giudice, R. Rattazzi and J. D. Wells, Nucl. Phys. B 595, 250 (2001) [arXiv:hep-ph/0002178].

[25] P. Creminelli and A. Strumia, Nucl. Phys. B 596, 125 (2001) [arXiv:hep$\mathrm{ph} / 0007267]$.

[26] S. Cullen, M. Perelstein and M. E. Peskin, Phys. Rev. D 62, 055012 (2000) [arXiv:hep-ph/0001166].

[27] G. F. Giudice and A. Strumia, Nucl. Phys. B 663, 377 (2003) [arXiv:hep$\mathrm{ph} / 0301232]$.

[28] S. B. Giddings and S. Thomas, Phys. Rev. D 65, 056010 (2002) [arXiv:hepph/0106219]; S. Dimopoulos and G. Landsberg, Phys. Rev. Lett. 87, 161602 (2001) [arXiv:hep-ph/0106295].

[29] S. Dimopoulos and R. Emparan, Phys. Lett. B 526, 393 (2002) [arXiv:hep$\mathrm{ph} / 0108060]$.

[30] G. F. Giudice, R. Rattazzi and J. D. Wells, Nucl. Phys. B 630, 293 (2002) [arXiv:hep-ph/0112161].

[31] V. A. Rubakov, Phys. Usp. 44, 871 (2001) [Usp. Fiz. Nauk 171, 913 (2001)] [arXiv:hep-ph/0104152].

[32] C. Csaki, arXiv:hep-ph/0404096.

[33] J. Maldacena, Adv. Theor. Math. Phys. 2 (1998) 231 [hep-th/9711200].

[34] S. S. Gubser, I. R. Klebanov and A. M. Polyakov, Phys. Lett. B428 (1998) 105 [hep-th/9802109]; E. Witten, Adv. Theor. Math. Phys. 2, 253 (1998) [hepth/9802150]. 
[35] W. D. Goldberger and M. B. Wise, Phys. Rev. Lett. 83 (1999) 4922 [hep$\mathrm{ph} / 9907447]$.

[36] R. Rattazzi and A. Zaffaroni, JHEP 0104, 021 (2001) [arXiv:hep-th/0012248].

[37] S. Coleman and E. Weinberg, Phys. Rev. D 7 (1973) 1888.

[38] N. Kaloper, Phys. Rev. D 60, 123506 (1999) [arXiv:hep-th/9905210].

[39] Z. Chacko and P. J. Fox, Phys. Rev. D 64, 024015 (2001) [arXiv:hepth/0102023].

[40] C. Csaki, M. Graesser, C. F. Kolda and J. Terning, Phys. Lett. B 462, 34 (1999)

[41] J. M. Cline, C. Grojean and G. Servant, Phys. Rev. Lett. 83, 4245 (1999)

[42] C. Csaki, M. Graesser, L. Randall and J. Terning, Phys. Rev. D 62, 045015 (2000) [arXiv:hep-ph/9911406].

[43] H. Davoudiasl, J. L. Hewett and T. G. Rizzo, Phys. Rev. Lett. 84, 2080 (2000) [arXiv:hep-ph/9909255].

[44] L. Randall and R. Sundrum, Phys. Rev. Lett. 83 (1999) 4690 [hep-th/9906064].

[45] A. Pomarol, Phys. Lett. B 486, 153 (2000) [arXiv:hep-ph/9911294].

[46] J. Maldacena, unpublished; E. Witten, Talk at ITP conference New Dimensions in Field Theory and String Theory, Santa Barbara http://www.itp.ucsb.edu/online/susy_c99/discussion; S. S. Gubser, "AdS/CFT and gravity," Phys. Rev. D 63, 084017 (2001) [hep-th/9912001]; H. Verlinde, "Holography and compactification," Nucl. Phys. B580, 264 (2000) [hep-th/9906182].

[47] N. Arkani-Hamed, M. Porrati and L. Randall, [hep-th/0012148]. 